\title{
Higher derivative scalar quantum field theory in curved spacetime
}

\author{
G. W. Gibbons $\odot,{ }^{1}$ C. N. Pope $\odot,{ }^{2,1}$ and Sergey Solodukhin ${ }^{3}$ \\ ${ }^{1}$ DAMTP, Centre for Mathematical Sciences, Cambridge University, Wilberforce Road, \\ Cambridge CB3 OWA, United Kingdom \\ ${ }^{2}$ Mitchell Institute for Fundamental Physics and Astronomy, Texas A\&M University, \\ College Station, Texas 77843-4242, USA \\ ${ }^{3}$ Institut Denis Poisson, UMR CNRS 7013, Université de Tours, Université d'Orléans, \\ Parc de Grandmont, 37200 Tours, France
}

(Received 12 September 2019; published 13 November 2019)

\begin{abstract}
We study a free scalar field $\phi$ in a fixed curved background spacetime subject to a higher derivative field equation of the form $F(\square) \phi=0$, where $F$ is a polynomial of the form $F(\square)=\prod_{i}\left(\square-m_{i}^{2}\right)$ and all masses $m_{i}$ are distinct and real. Using an auxiliary field method to simplify the calculations, we obtain expressions for the Belinfante-Rosenfeld symmetric energy-momentum tensor and compare it with the canonical energy-momentum tensor when the background is Minkowski spacetime. We also obtain the conserved symplectic current necessary for quantization and briefly discuss the issue of negative energy vs negative norm and its relation to reflection positivity in Euclidean treatments. We study, without assuming spherical symmetry, the possible existence of finite energy static solutions of the scalar equations, in static or stationary background geometries. Subject to various assumptions on the potential, we establish nonexistence results including a no-scalar-hair theorem for static black holes. We consider Pais-Uhlenbeck field theories in a cosmological de Sitter background and show how the Hubble friction may eliminate what would otherwise be unstable behavior when interactions are included.
\end{abstract}

DOI: $10.1103 /$ PhysRevD.100.105008

\section{INTRODUCTION}

Higher derivative field theories have received a considerable amount of attention over the years for a variety of reasons, not least because of the realization that theories incorporating standard Einstein gravity inevitably suffer from problems of non-renormalizability. Although it was shown by 't Hooft and Veltman [1] that pure Einstein gravity itself was finite at one loop, this does not persist at higher loop order [2], nor if (generic) matter is included. The demonstration by Stelle [3] in the 1970s that the nonrenormalizability problem itself could be overcome by adding quadratic curvature terms to the action opened the door to many investigations of such theories, although the realization that renormalizability could only thereby be achieved at the price of introducing ghost states of negative norm, or energies that are unbounded below, dampened the enthusiasm for the idea. Subsequently, with the development of string theory, the idea of higher derivative corrections to gravity acquired a new impetus, but now

Published by the American Physical Society under the terms of the Creative Commons Attribution 4.0 International license. Further distribution of this work must maintain attribution to the author(s) and the published article's title, journal citation, and DOI. Funded by SCOAP . in the framework of an effective field theory in which quadratic curvature corrections would represent just the start of an infinite sequence of higher-order terms. Within this framework, a focus on any particular lower-order term or terms in the infinite sequence of corrections would seem to be unjustified, since in circumstances where such a term or terms leads to substantial modifications to the solutions or spectrum of the theory, yet-higher order terms that are being ignored would have at least as important an effect.

Although the conviction that the ghost states or unbounded negative energies of a finite-order higher derivative theory are sufficient to rule them out of consideration is widespread, it is perhaps still worthwhile to study them in more detail, and to investigate whether the ostensible problems really are as severe as is commonly believed. One of the earliest investigations of higher derivative field theories was in the classic work of Pais and Uhlenbeck [4], who studied scalar field theories in Minkowski spacetime. Much of their focus was on differential operators of infinite order, such as the exponential of the d'Alembertian, but they did also consider operators of finite order, such as powers of the d'Alembertian or products of massive Klein-Gordon operators. Many of the features that are encountered in theories such as these will have counterparts in more complicated theories such as higher derivative theories of gravity. 
As a preliminary to studying higher derivative field theories, much insight can be gained in the simpler case of a classical higher derivative particle mechanical theory and its quantum mechanical extension. A simple and much studied example is the Pais-Uhlenbeck oscillator, $\left(D^{2}-\omega_{1}^{2}\right)$ $\left(D^{2}-\omega_{2}^{2}\right) x(t)=0$, where $D=d / d t$. Its Hamiltonian, constructed using the Ostrogradsky procedure [5], which corresponds essentially to the difference between standard oscillator Hamiltonians for particles with frequencies $\omega_{1}$ and $\omega_{2}$, is unbounded both above and below. At the level of the free theory this is not a problem, but a widespread intuition is that once interactions are included, instabilities will inevitably set in since the energies in the negative energy modes and the positive energy modes can both increase (in magnitude) while the total energy remains constant. ${ }^{1}$ A number of studies have shown that this need not, in fact, be the case [6-11]. For example, for given initial conditions, an added $\lambda x^{4}$ interaction term will not give instabilities provided $\lambda$ is sufficiently small [6]. And if one instead adds a bounded potential, such as $\lambda \sin ^{4} x$, the evolution of initial data is unconditionally stable [6]. For a consideration of the Pais-Uhlenbeck oscillator from the point of view of statistical physics, see [12].

It has often been argued (see, for example, Ref. [13]) that instability problems in a higher derivative field theory will be much more severe than in a particle mechanical theory, because of the diverging entropy associated with the infinity of unobserved high-momentum states, and that this would lead to an instantaneous decay of the vacuum. Countering this, it has been argued that the divergent entropy results from integrating over the phase space of unobserved decay products, and that in a closed system such as our universe there exist no external observers who would integrate over this infinite phase space. Actual observers, inside the universe, would implicitly have measured the momenta of the particles, and so there would be no diverging phase space integrals leading to instantaneous vacuum decay [14]. It has, further, been argued that the experience with adding interactions to a higher derivative particle mechanics model, where potentials that are bounded both above and below do not give rise to any instabilities, suggests that similarly bounded potentials in a higher derivative field theory may not in any case give rise to instabilities [14]. There have also been proposals, such as that by Hawking [15], that one might impose boundary conditions to eliminate ghost excitations, at least in asymptotic states, since the concomitant acausality could be at sufficiently short timescales that it may not be observable in a Wheeler-DeWitt framework. More recently,

\footnotetext{
${ }^{1}$ Although this intuition is commonly attributed to Ostrogradsky, either as the "Ostrogradsky instability" or "Ostrogradsky's instability theorem," it appears that Ostrogradsky himself never actually discussed the question of instabilities in higher derivative theories.
}

Hawking and Hertog [16] argued that in a path-integral treatment in a Euclidean framework, one can obtain welldefined rules for calculating probabilities, after continuing to Lorentzian spacetime, even in a higher derivative field theory.

In the light of some of these investigations, we shall take the view that it is still of considerable interest to study the properties of higher derivative field theories, and that scalar theories of this kind may be useful as models for more complicated systems such as higher derivative gravities. Most of the previous work on higher derivative scalar field theories has been in a flat Minkowski spacetime background. Our main purpose, in this paper, is to consider scalar theories of the kind studied by Pais and Uhlenbeck, but now within the framework of curved spacetime backgrounds. We shall show how the calculation of the Belinfante-Rosenfeld energy-momentum tensor can be greatly simplified by introducing an auxiliary field formalism, and we shall also make comparisons with the canonical flat spacetime energy-momentum tensor calculated using Noether methods. (An action was constructed using an auxiliary field formulation in [17], although it was not employed in order to calculate an energy-momentum tensor.) We also obtain some general results for the symplectic Noether currents that can be used in order to construct the norms on states in the theories. One further motivation for studying higher derivative scalar field theories in curved backgrounds is that, as we shall discuss later, this can actually help to mitigate some of the instabilities that might otherwise occur in flat spacetime.

The organization of the paper is as follows. We begin in Sec. II with a review of some basic aspects of the classical and quantum properties of a point particle moving in one dimension, governed by a higher derivative equation of motion. Our discussion includes a review of the Ostrogradsky $[5,18]$ construction of the Hamiltonian describing the system, and an elegant analysis by Smilga [9] of the quantum theory of a fourth-order Pais-Uhlenbeck oscillator. In Sec. III we review some properties of a scalar Pais-Uhlenbeck field theory in a Minkowski background, including the construction of the canonical energymomentum tensor and the conserved symplectic current. In Sec. IV we extend our discussion to the scalar PaisUhlenbeck field theory in a curved spacetime background, showing how one can use an auxiliary field formulation in order to facilitate the construction of the BelinfanteRosenfeld energy-momentum tensor, which allows a consistent coupling to the gravitational field. By this means, one avoids the necessity of varying the metrics in high powers of the covariant derivative.

In Sec. V we discuss the quantization of higher derivative scalar field theories using Euclidean methods. This may be done if the background metric admits an analytic continuation containing a real section on which the metric is positive definite, as it does for real tunneling metrics [19]. 
It may also arise in a full blown Euclidean quantum gravity path integral calculation when a gravitational instanton saddle point admits a reflection map, i.e., an involutive isometry fixing a separating hypersurface. We generalize previous arguments [15] showing that if one adopts the Osterwalder and Schrader prescription [20] for constructing the quantum mechanical Hilbert space, then reflection positivity is not satisfied despite the Euclidean action of the scalar field being positive definite. In other words, negative norm states are inevitable.

In Sec. VI, subject to various assumptions about the scalar potential, if present, we prove nonexistence results for static solutions of the equations of motion in static or stationary spacetimes. The background could be Minkowski spacetime, in which case these results rule out stable solitons and unstable sphaleron type solutions. They also hold in globally stationary backgrounds. They are easily extended to cover the case of static black hole backgrounds with regular event horizons.

In Sec. VII, we consider Pais-Uhlenbeck field theories in a de Sitter spacetime background and show how Hubble friction can eliminate instabilities that would otherwise occur when nonlinear couplings are present. These examples serve to illustrate the fact that the often-claimed instabilities of higher-order field theories can sometimes be illusory.

\section{A HIGHER DERIVATIVE POINT PARTICLE}

To orient ourselves, we begin by recalling some facts about theories of particles moving in one spatial dimension governed by a Lagrangian $L=L(x, \dot{x}, \ddot{x}, t)$ depending upon the position $x$, velocity $\dot{x}$, and acceleration $\ddot{x}$. Varying the Lagrangian gives

$$
\begin{aligned}
\delta L-\delta t \frac{\partial L}{\partial t}= & \delta x \frac{\partial L}{\partial x}+\delta \dot{x} \frac{\partial L}{\partial \dot{x}}+\delta \ddot{x} \frac{\partial L}{\partial \ddot{x}} \\
= & \delta x\left(\frac{\partial L}{\partial x}-\frac{d}{d t} \frac{\partial L}{\partial \dot{x}}\right)+\frac{d}{d t}\left(\delta x \frac{\partial L}{\partial \dot{x}}\right) \\
& -\delta \dot{x} \frac{d}{d t} \frac{\partial L}{\partial \ddot{x}}+\frac{d}{d t}\left(\delta \dot{x} \frac{\partial L}{\partial \ddot{x}}\right) \\
= & \delta x\left(\frac{\partial L}{\partial x}-\frac{d}{d t} \frac{\partial L}{\partial \dot{x}}+\frac{d^{2}}{d t^{2}} \frac{\partial L}{\partial \ddot{x}}\right) \\
& +\frac{d}{d t}\left(\delta x \frac{\partial L}{\partial \dot{x}}+\delta \dot{x} \frac{\partial L}{\partial \ddot{x}}-\delta x \frac{d}{d t} \frac{\partial L}{\partial \ddot{x}}\right) .
\end{aligned}
$$

The equation of motion is thus

$$
\frac{\partial L}{\partial x}-\frac{d}{d t} \frac{\partial L}{\partial \dot{x}}+\frac{d^{2}}{d t^{2}} \frac{\partial L}{\partial \ddot{x}}=0 .
$$

\section{A. Energy and momentum conservation}

If the equation of motion holds, then

$$
\frac{d}{d t}\left(L-\dot{x} \frac{\partial L}{\partial \dot{x}}-\ddot{x} \frac{\partial L}{\partial \ddot{x}}+\dot{x} \frac{d}{d t} \frac{\partial L}{\partial \ddot{x}}\right)=\frac{\partial L}{\partial t} .
$$

Thus, if $L$ does not depend upon $t$, there is a conserved energy

$$
E=\dot{x} \frac{\partial L}{\partial \dot{x}}+\ddot{x} \frac{\partial L}{\partial \ddot{x}}-\dot{x} \frac{d}{d t} \frac{\partial L}{\partial \ddot{x}}-L,
$$

which may be written as

$$
E=\dot{x}\left(\frac{\partial L}{\partial \dot{x}}-\frac{d}{d t} \frac{\partial L}{\partial \ddot{x}}\right)+\ddot{x} \frac{\partial L}{\partial \ddot{x}}-L .
$$

If the Lagrangian is translationally invariant, $\frac{\partial L}{\partial x}=0$, we expect that momentum should be conserved. With an eye on the equation of motion (2.4), we define the momentum $p_{x}$ by

$$
p_{x}=\frac{\partial L}{\partial \dot{x}}-\frac{d}{d t} \frac{\partial L}{\partial \ddot{x}},
$$

so that equation of motion (2.4) takes the form

$$
\dot{p}_{x}=\frac{\partial L}{\partial x}
$$

and hence if the Lagrangian is translationally invariant, the momentum $p_{x}$ is conserved. In terms of $p_{x}$, the energy is given by

$$
E=\dot{x} p_{x}+\ddot{x} \frac{\partial L}{\partial \ddot{x}}-L .
$$

\section{B. Ostrogradsky's Hamiltonian}

Following $[4,5,18]{ }^{2}$ we define

$$
y=\dot{x}, \quad p_{y}=\frac{\partial L}{\partial \ddot{x}} .
$$

If the "nondegeneracy" condition

$$
\frac{\partial^{2} L}{\partial \ddot{x}^{2}} \neq 0
$$

holds, then we may solve for the acceleration as a function of $\left(x, y, p_{y}\right)$ :

$$
\ddot{x}=a\left(x, y, p_{y}\right) .
$$

\footnotetext{
${ }^{2}$ For a geometrical treatment, see [21].
} 
To obtain the Hamiltonian, we take the Legendre transform of the Lagrangian and obtain

$H=p_{x} y+p_{y} a\left(x, y, p_{y}\right)-L\left(x, y, a\left(x, y, p_{y}\right)\right)$.

As a concrete example, consider the Pais-Uhlenbeck oscillator, described by the Lagrangian

$$
L=\frac{1}{2} \dot{x}^{2}-\frac{1}{2} \beta \ddot{x}^{2}-\frac{1}{2} \Omega^{2} x^{2} .
$$

Clearly the nondegeneracy condition holds as long as $\beta \neq 0$, since

$$
\frac{\partial^{2} L}{\partial \ddot{x}^{2}}=-\beta
$$

The equations of motion are

$$
\ddot{x}+\beta \dddot{x}+\Omega^{2} x=0,
$$

i.e.,

$$
p_{y}=-\beta \ddot{x} \quad \Rightarrow \quad \ddot{x}=-\frac{1}{\beta} p_{y}=a \text {. }
$$

We have

$$
p_{x}=\dot{x}+\beta \dddot{x},
$$

and so the equation of motion implies

$$
\dot{p}_{x}=-\Omega^{2} x .
$$

In a general potential $V(x)$ we would have

$$
\dot{p}_{x}=-\frac{d V}{d x} .
$$

Thus Newton's second law, expressed in terms of momentum, is obeyed.

Making the ansatz $x=\Re A e^{-i \omega t}$ we find

$$
\omega^{4}-\frac{1}{\beta} \omega^{2}+\frac{\Omega^{2}}{\beta}=0,
$$

and thus

$$
\omega^{2}=\frac{1}{2 \beta}\left(1 \pm \sqrt{1-4 \beta \Omega^{2}}\right) .
$$

If $0<4 \beta \Omega^{2}<1$, then all four roots $\omega=\left( \pm \omega_{1}, \pm \omega_{2}\right)$ will be real, distinct, and nonvanishing. Useful relations are

$$
\omega_{1}^{2}+\omega_{2}^{2}=\frac{1}{\beta}, \quad \omega_{1}^{2} \omega_{2}^{2}=\frac{\Omega^{2}}{\beta} .
$$

The equation of motion may be written in terms of $\omega_{1}$ and $\omega_{2}$ as

$$
\dddot{x}+\left(\omega_{1}^{2}+\omega_{2}^{2}\right) \ddot{x}+\omega_{1}^{2} \omega_{2}^{2} x=0 .
$$

We write the general solution as

$$
x=A e^{-i \omega_{1} t}+B e^{-i \omega_{2} t}+\bar{A} e^{i \omega_{1} t}+\bar{B} e^{i \omega_{2} t},
$$

and therefore

$$
\begin{aligned}
x(0) & =(A+\bar{A})+(B+\bar{B}), \\
\dot{x}(0) & =-i \omega_{1}(A-\bar{A})-i \omega_{2}(B-\bar{B}), \\
\ddot{x}(0) & =-\omega_{1}^{2}(A+\bar{A})-\omega_{2}^{2}(B+\bar{B}), \\
\dddot{x}(0) & =i \omega_{1}^{3}(A-\bar{A})+i \omega_{2}^{3}(B-\bar{B}) .
\end{aligned}
$$

Since $\omega_{1} \neq \omega_{2}$, there exists a unique solution for $A$ and $B$ for all initial data $[x(0), \dot{x}(0), \ddot{x}(0)$, and $\dddot{x}(0)]$. Moreover, this solution is bounded for all time.

Thus the theory is predictive, the initial value problem has a solution for all time, and it is well posed: it depends continuously on the initial data.

It is, however, true that the conserved energy

$$
E=\frac{1}{2}(\dot{x})^{2}-\frac{1}{2} \beta(\ddot{x})^{2}+\beta \dot{x} \dddot{x}+\frac{1}{2} \Omega^{2} x^{2},
$$

or equivalently in terms of the canonically conjugate variables, the Hamiltonian

$$
\begin{aligned}
H & =p_{x} y-\frac{p_{y}^{2}}{2 \beta}-\frac{1}{2} y^{2}+\frac{1}{2} \Omega^{2} x^{2} \\
& =\frac{1}{2} p_{x}^{2}+\frac{1}{2} \Omega^{2} x^{2}-\frac{1}{2}\left(y-p_{x}\right)^{2}+\frac{p_{y}^{2}}{2 \beta}
\end{aligned}
$$

depends linearly on $p_{x}$, and it is the sum of two positive and two negative squares (if $\beta$ is positive, as discussed above). In fact, Hamilton's equations

$$
\begin{aligned}
& \dot{p}_{x}=-\Omega^{2} x, \quad \dot{p}_{y}=-p_{x}+y, \\
& \dot{x}=y, \quad \dot{y}=-\frac{p_{y}}{\beta}
\end{aligned}
$$

in this case are linear, and one may verify that they have the same characteristic frequencies.

As mentioned in the Introduction, it is less obvious what happens if an interaction term is added, rendering the equations of motion nonlinear. An example would be a potential term of the form $\lambda x^{4}$, where $\lambda$ is a constant. A widely held intuition is that some sort of instability 
will result, but, as shown in [6], for given initial conditions the evolution may remain bounded if the $\lambda$ interaction is sufficiently small. The question may also depend crucially on the definition of stability. The motion may always remain within a bounded neighborhood of the unperturbed motion, $x=0$ for example, even though it may not return asymptotically to $x=0$. Moreover the timescale for any instability may, in the cosmological context, be sufficiently long, for small enough $\lambda$, as not to lead to any observable effect within the relevant timescale.

\section{Quantization of the Pais-Uhlenbeck oscillator}

An obvious procedure would be to consider wave functions of the form $\Psi=\Psi(x, y)$, in which case

$$
\hat{p}_{x}=-i \frac{\partial}{\partial x}, \quad \hat{p}_{y}=-i \frac{\partial}{\partial y},
$$

leading to

$$
\hat{H}=-i y \frac{\partial}{\partial x}-\frac{1}{2} \frac{\partial^{2}}{\partial y^{2}}-\frac{1}{2} y^{2}+\frac{1}{2} \Omega^{2} x^{2}
$$

More revealing would be to consider wave functions of the form $\Psi=\Psi\left(x, p_{y}\right)$ in which case

$$
\hat{p}=-i \frac{\partial}{\partial x}, \quad \hat{y}=i \frac{\partial}{\partial p_{y}},
$$

leading to

$$
\hat{H}=\frac{\partial^{2}}{\partial x \partial y}-\frac{1}{2} \frac{\partial^{2}}{\partial p_{y}^{2}}-\frac{p_{y}^{2}}{2 \beta}+\frac{1}{2} \Omega^{2} x^{2} .
$$

The first two terms are the wave equations in twodimensional Minkowski spacetime with the flat metric

$$
d s^{2}=d x^{2}+2 d x d p_{y}=\left(d x+d p_{y}\right)^{2}-d p_{y}^{2},
$$

in which $x$ is a lightlike coordinate.

Smilga [9] succeeded in finding a canonical transformation that clarifies what is going on. Starting with the (rescaled) Lagrangian

$$
L=\frac{1}{2}\left[(\ddot{x})^{2}-\left(\omega_{1}^{2}+\omega_{2}^{2}\right)(\dot{x})^{2}+\omega_{1}^{2} \omega_{2}^{2} x^{2}\right],
$$

Ostrogradsky's Hamiltonian becomes

$$
H=p_{x} y+\frac{1}{2} p_{y}^{2}+\frac{1}{2}\left(\omega_{1}^{2}+\omega_{2}^{2}\right) y^{2}-\frac{1}{2} \omega_{1}^{2} \omega_{2}^{2} x^{2} .
$$

Now let

$$
\begin{aligned}
& x=\frac{1}{\omega_{1}} \frac{\omega_{1} Y-p_{X}}{\sqrt{\omega_{1}^{2}-\omega_{2}^{2}}}, \quad p_{x}=\omega_{1} \frac{\omega_{1} p_{Y}-\omega_{2}^{2} X}{\sqrt{\omega_{1}^{2}-\omega_{2}^{2}}}, \\
& y=\frac{\omega_{1} X-p_{Y}}{\sqrt{\omega_{1}^{2}-\omega_{2}^{2}}}, \quad p_{y}=\frac{\omega_{1} p_{X}-\omega_{2}^{2} Y}{\sqrt{\omega_{1}^{2}-\omega_{2}^{2}}},
\end{aligned}
$$

which implies

$$
\begin{array}{rlrl}
X & =\frac{1}{\omega_{1}} \frac{p_{x}+\omega_{1}^{2} y}{\sqrt{\omega_{1}^{2}-\omega_{2}^{2}}}, & Y=\frac{p_{y}+\omega_{1}^{2} x}{\sqrt{\omega_{1}^{2}-\omega_{2}^{2}}}, \\
p_{X}=\omega_{1} \frac{p_{y}+\omega_{2}^{2} x}{\sqrt{\omega_{1}^{2}-\omega_{2}^{2}}}, & p_{Y}=\frac{p_{x}+\omega_{2}^{2} y}{\sqrt{\omega_{1}^{2}-\omega_{2}^{2}}} .
\end{array}
$$

This is a canonical transformation, since

$$
d p_{x} \wedge d x+d p_{y} \wedge d y=d p_{X} \wedge d X+d p_{Y} \wedge d Y .
$$

Pulling back the Hamiltonian one finds

$$
H=\frac{1}{2}\left(p_{X}^{2}+\omega_{1}^{2} X^{2}\right)-\frac{1}{2}\left(p_{Y}^{2}+\omega_{2}^{2} Y^{2}\right) .
$$

This may be quantized in the obvious way, with a positive norm on the Hilbert space, by introducing annihilation and creation operators in the standard way, with the nonvanishing commutators $\left[a_{X}, a_{X}^{\dagger}\right]=\left[a_{Y}, a_{Y}^{\dagger}\right]=1$. We have

$$
\hat{H}=\left(\hat{a}_{X}^{\dagger} \hat{a}_{X}+\frac{1}{2}\right) \omega_{1}-\left(\hat{a}_{Y}^{\dagger} \hat{a}_{Y}+\frac{1}{2}\right) \omega_{2} .
$$

Assuming that the "ground state" satisfies

$$
\hat{a}_{X}|0\rangle=\hat{a}_{Y}|0\rangle=0,
$$

all norms are positive, but the spectrum of the Hamitonian is unbounded below.

Alternatively, we could assume that the vacuum is defined by

$$
\hat{a}_{X}|0\rangle=\hat{a}_{Y}^{\dagger}|0\rangle=0,
$$

in which case the spectrum of the Hamiltonian is bounded below but the norm on states would be indefinite. For example, the state $a_{Y}|0\rangle$ now has the norm

$$
\left.\left|a_{Y}\right| 0\right\rangle\left.\right|^{2}=\left\langle 0\left|a_{Y}^{\dagger} a_{Y}\right| 0\right\rangle=-\langle 0 \mid 0\rangle .
$$

(For different approaches entailing the introduction of nonstandard time inversion parity assignments, see $[22,23]$.) 


\section{PAIS-UHLENBECK FIELD THEORY IN MINKOWSKI SPACETIME}

In this section we recall some aspects of the simplest noninteracting higher derivative scalar field theories in a Minkowski background spacetime. The field equations are of the form [4]

$$
E \phi \equiv F(\square) \phi=0,
$$

where ${ }^{3}$

$$
\square=-\partial_{t}^{2}+\nabla^{2}=\eta^{\mu \nu} \partial_{\mu} \partial_{\nu}, \quad \partial_{\mu}=\left(\partial_{t}, \partial_{x^{i}}\right),
$$

and $F(u)$ is a real valued function of $u$.

A suitable action for a real scalar $\phi$, up to factors and possible boundary terms, is

$$
\frac{1}{2} \int d^{4} x \phi F(\square) \phi
$$

The Lagrangian models studied by Ostrogradsky are obtained by replacing $\square \phi$ in (3.1) by $-D^{2}$, where $D=\frac{d}{d t}$.

A simple nontrivial example is

$$
E \phi=\left(\square-m_{1}^{2}\right)\left(\square-m_{2}^{2}\right) \phi=0,
$$

where, unless otherwise stated, we assume that the masses $m_{1}$ and $m_{2}$ are real and distinct. The case when $m_{1}^{2}=m_{2}^{2}$ has some different properties, and it is referred to as dipolar. If $\square=-D^{2}$ we obtain the so-called Pais-Uhlenbeck oscillator.

As we shall see, one may always introduce a symplectic current $J^{\mu}\left(\phi_{1}, \phi_{2}\right)=-J^{\mu}\left(\phi_{2}, \phi_{1}\right)$ such that

$$
\phi_{1} F(\square) \phi_{2}-\phi_{2} F(\square) \phi_{2}=\partial_{\mu} J^{\mu}\left(\phi_{1}, \phi_{2}\right) \text {. }
$$

Thus on shell,

$$
\partial_{\mu} J^{\mu}=0,
$$

and hence a conserved (pre)symplectic form on solutions $\phi_{1}$ and $\phi_{2}$ of (3.1) is given by

$$
\omega\left(\phi_{1}, \phi_{2}\right)=-\omega\left(\phi_{2}, \phi_{1}\right)=\int_{\Sigma} J^{\mu} d \Sigma_{\mu},
$$

where $\Sigma$ is any Cauchy surface. (The prefix "pre" would apply if the symplectic form were degenerate.)

The equation of motion (3.1) is clearly linear and if $F(\square)$ is a polynomial of degree $N$, then of necessity quasilinear, ${ }^{4}$ and hence its characteristic surfaces $S=$ const must, by standard theory, satisfy

\footnotetext{
${ }^{3}$ Throughout the paper we adhere to the same -+++ signature convention as [4].

${ }^{4}$ In other words, all highest derivative terms occur linearly.
}

$$
\left(\eta^{\mu \nu} \partial_{\mu} S \partial_{\nu} S\right)^{N}=0
$$

In other words, the characteristic surfaces are null hypersurfaces. Thus (3.1) satisfies the Einstein-causality principle.

Since (3.1) is linear we may substitute in it the Ansatz

$$
\phi=A e^{i k_{\nu} x^{\mu}}=A e^{i(-\omega t+\mathbf{k} \cdot \mathbf{x})},
$$

where $A$ and $k_{\mu}$ are constant, and hence we find that

$$
F\left(-k_{\mu} k^{\mu}\right)=0,
$$

where $k_{\mu}=(-\omega, \mathbf{k})$. If the zeros of $F(u)$ are at $u=m_{i}^{2}$ with real $m_{i}$, then we have several branches to the dispersion relation:

$$
\omega= \pm \sqrt{m_{i}^{2}+\mathbf{k}^{2}}
$$

\section{A. The energy momentum tensor}

In the simple case (3.4), an energy-momentum tensor is presented in [24]. Making allowance for the conversion from the $(+---)$ spacetime signature used in that reference, and also our choice for the overall sign for the Pais-Uhlenbeck action, this symmetric tensor is [24]

$$
\begin{aligned}
\bar{T}^{\mu \nu}= & 2\left(\partial^{\mu} \partial^{\nu} \phi\right) \square \phi-\left(\partial^{\mu} \phi\right) \square\left(\partial^{\nu} \phi\right)-\left(\partial^{\nu} \phi\right) \square\left(\partial^{\mu} \phi\right) \\
& +\left(\partial^{\mu} \partial^{\nu} \partial_{\alpha} \phi\right)\left(\partial^{\alpha} \phi\right)-\left(\partial^{\mu} \partial^{\alpha} \phi\right)\left(\partial^{\nu} \partial_{\alpha} \phi\right) \\
& -\frac{1}{2} \eta^{\mu \nu}(\square \phi)^{2}-\frac{1}{2}\left(m_{1}^{2}+m_{2}^{2}\right) \\
& \times\left(\phi \partial^{\mu} \partial^{\nu} \phi-\partial^{\mu} \phi \partial^{\nu} \phi-\eta^{\mu \nu} \phi \square \phi\right) \\
& -\frac{1}{2} \eta^{\mu \nu} m_{1}^{2} m_{2}^{2} \phi^{2} .
\end{aligned}
$$

It obeys

$$
\partial_{\mu} \bar{T}^{\mu \nu}=-\left(\partial^{\nu} \phi\right) E \phi,
$$

and thus is conserved on shell. Moreover if $\phi=\phi(t)$, then

$\bar{T}^{00}=-\frac{1}{2} \ddot{\phi}^{2}+\dot{\phi} \dddot{\phi}+\frac{1}{2}\left(m_{1}^{2}+m_{2}^{2}\right) \dot{\phi}^{2}+\frac{1}{2} m_{1}^{2} m_{2}^{2} \phi^{2}$,

which agrees with the energy of the Pais-Uhlenbeck oscillator derived using Noether's theorem.

The derivative terms in (3.12) differ from those in the standard energy-momentum tensor for a scalar field that one obtains by the Belinfante-Rosenfeld $[25,26]$ procedure (i.e., minimally coupling the action to a metric $g_{\mu \nu}$ and taking the variational derivative). Later we shall construct an energy-momentum tensor using the Belinfante-Rosenfeld 
procedure, and in Appendix we show how it is related to (3.12).

\section{B. Second quantization of the Pais-Uhlenbeck field theory}

Recall that for a free theory we first construct the oneparticle Hilbert space and then construct the full (nonseparable) Hilbert space using the Fock construction. The standard approach is to complexify the Cauchy data and introduce a (symmetric) sesquilinear form

$$
h\left(\phi_{1}, \phi_{2}\right)=-i \omega\left(\bar{\phi}_{1}, \phi_{2}\right),
$$

where now $\phi_{1}$ and $\phi_{2}$ are complex valued and

$$
\overline{h\left(\phi_{1}, \phi_{2}\right)}=h\left(\phi_{2}, \phi_{1}\right) \text {. }
$$

In the case of just two time derivatives in the equations of motion one has

$$
h\left(\phi_{1}, \phi_{2}\right)=-i \int_{E^{3}}\left(\dot{\bar{\phi}}_{1} \phi_{2}-\bar{\phi}_{1} \dot{\phi}_{2}\right) d^{3} x .
$$

If $\phi_{1}=\phi_{2}=A e^{-i \omega t}$, we have

$$
h(\phi, \phi)=2 \omega \int_{E^{3}}|A|^{2} d^{3} x .
$$

Thus positive norm states have $\omega>0$, and so we identify the one-particle Hilbert space with the space of positive frequency Cauchy data.

\section{Symplectic current for the Pais-Uhlenbeck field theory}

When $m_{1}^{2} \neq m_{2}^{2}$, the general solution of the PaisUhlenbeck equation $\left(\square-m_{1}^{2}\right)\left(\square-m_{2}^{2}\right) \phi=0$ is given by

$$
\phi=a \chi_{1}+b \chi_{2}
$$

where $\chi_{1}$ and $\chi_{2}$ are solutions of $\left(\square-m_{1}^{2}\right) \chi_{1}=0$ and $\left(\square-m_{2}^{2}\right) \chi_{2}=0$, and $a$ and $b$ are constants. Thus $\chi_{1}$ and $\chi_{2}$ provide a basis for the general solution. Without loss of generality, we shall assume $m_{1}^{2}>m_{2}^{2}$ in what follows.

We now write the field equation as

$$
\square^{2} \phi-\left(m_{1}^{2}+m_{2}^{2}\right) \square \phi+m_{1}^{2} m_{2}^{2} \phi=0 .
$$

In Minkowski spacetime we have the following identities:

$$
\begin{aligned}
J_{\square}^{\mu} & :=\phi_{1} \partial^{\mu} \phi_{2}-\left(\phi_{1} \leftrightarrow \phi_{2}\right), \\
\partial_{\mu} J_{\square}^{\mu} & =\phi_{1} \square \phi_{2}-\left(\phi_{1} \leftrightarrow \phi_{2}\right), \\
J_{\square^{2}}^{\mu} & :=\phi_{1} \partial^{\mu} \square \phi_{2}+\square \phi_{1} \partial^{\mu} \phi_{2}-\left(\phi_{1} \leftrightarrow \phi_{2}\right), \\
\partial_{\mu} J_{\square^{2}}^{\mu} & =\phi_{1} \square^{2} \phi_{2}-\left(\phi_{1} \leftrightarrow \phi_{2}\right) .
\end{aligned}
$$

Thus if

$$
J^{\mu}=J_{\square^{2}}^{\mu}-\left(m_{1}^{2}+m_{2}^{2}\right) J_{\square}^{\mu},
$$

then on shell

$$
\partial_{\mu} J^{\mu}=0
$$

Now suppose that $\phi_{1}$ and $\phi_{2}$ are any two solutions of

$$
\square \phi_{1}=\mu_{1}^{2} \phi_{1}, \quad \square \phi_{2}=\mu_{2}^{2} \phi_{2},
$$

where each of $\mu_{1}^{2}$ and $\mu_{2}^{2}$ can be either $m_{1}^{2}$ or $m_{2}^{2}$. Then

$$
\begin{gathered}
J^{\mu}=\left(\left(\mu_{1}^{2}+\mu_{2}^{2}\right)-\left(m_{1}^{2}+m_{2}^{2}\right)\right)\left(\phi_{1} \partial^{\mu} \phi_{2}-\phi_{2} \partial^{\mu} \phi_{1}\right) \\
=\left(\left(\mu_{1}^{2}+\mu_{2}^{2}\right)-\left(m_{1}^{2}+m_{2}^{2}\right)\right) J_{\square}^{\mu} .
\end{gathered}
$$

Evidently if $\phi_{1}$ and $\phi_{2}$ have different masses (i.e., if $\mu_{1}^{2}=$ $m_{1}^{2}$ and $\mu_{2}^{2}=m_{2}^{2}$, or else if $\mu_{1}^{2}=m_{2}^{2}$ and $\mu_{2}^{2}=m_{1}^{2}$ ), then $J^{\mu}=0$.

If $\phi_{1}$ and $\phi_{2}$ have equal mass-squared $\mu_{1}^{2}=\mu_{2}^{2}=m_{1}^{2}$, then

$$
J^{\mu}=\left(m_{1}^{2}-m_{2}^{2}\right) J_{\square}^{\mu} .
$$

while if $\phi_{i}$ and $\phi_{2}$ have equal mass-squared $\mu_{1}^{2}=\mu_{2}^{2}=m_{2}^{2}$, then

$$
J^{\mu}=\left(m_{2}^{2}-m_{1}^{2}\right) J_{\square}^{\mu} .
$$

If we choose the complex modes with mass $m_{1}$ to have positive frequency and the complex modes with mass $m_{2}$ to have negative frequency, the Hermitian form $h(\cdot, \cdot)$ will be positive definite and diagonalized in this basis.

Thus, despite the fact that we associate one creation operator with a positive frequency mode and the other with a negative frequency mode, we obtain a positive norm on our Hilbert space. There are no states with negative norm (sometimes called "ghosts"). Of course, if $m_{2}^{2}>m_{1}^{2}$, we must reverse the convention.

\section{PAIS-UHLENBECK FIELD THEORY IN CURVED SPACETIME}

\section{A. Simplest model}

We now replace $\partial_{\mu}$ by $\nabla_{\mu}$, the Levi-Civita covariant derivative, and so $\square=\nabla_{\mu} \nabla^{\mu}$, the generally covariant d'Alembertian. The field equation is

$$
\left.E \phi \equiv\left(\square^{2}-\left(m_{1}^{2}+m_{2}^{2}\right) \square+m_{1}^{2} m_{2}^{2}\right)\right) \phi=0 .
$$

The field equation may be derived from the action 


$$
\begin{aligned}
S= & -\frac{1}{2} \int \sqrt{-g} d^{4} x\left[(\square \phi)^{2}+\left(m_{1}^{2}+m_{2}^{2}\right) g^{\mu \nu} \partial_{\mu} \phi \partial_{\nu} \phi\right. \\
& \left.+m_{1}^{2} m_{2}^{2} \phi^{2}\right],
\end{aligned}
$$

from which one finds that under a variation of $\phi$,

$$
\begin{aligned}
\delta S= & -\int_{D} \sqrt{-g} d^{4} x \delta \phi E \phi+\int_{\partial D}\left(\nabla^{\mu} \square \phi \delta \phi-\square \phi \nabla^{\mu} \delta \phi\right. \\
& \left.-\left(m_{1}^{2}+m_{2}^{2}\right) \nabla^{\mu} \phi \delta \phi\right) d \Sigma_{\mu} .
\end{aligned}
$$

The operator $E$ may also be written as

$$
E=\left(\square-m_{1}^{2}\right)\left(\square-m_{2}^{2}\right)=\left(\square-m_{2}^{2}\right)\left(\square-m_{1}^{2}\right) .
$$

The identities

$$
\begin{gathered}
\chi \square \phi=\nabla_{\mu}\left(\chi \nabla^{\mu} \phi\right)-g_{\mu \nu} \nabla^{\mu} \chi \nabla^{\nu} \phi, \\
\chi \square^{2} \phi=\nabla_{\mu}\left(\chi \nabla^{\mu} \square \phi-\left(\nabla^{\mu} \chi\right) \square \phi\right)+(\square \chi)(\square \phi),
\end{gathered}
$$

imply the formal self-adjointness of $E$ since, from them, one deduces that

$$
\chi E \phi-\phi E \chi=\nabla_{\mu} J^{\mu},
$$

where

$$
\begin{aligned}
J^{\mu}[\chi, \phi]= & \chi \nabla^{\mu} \square \phi-\left(\nabla^{\mu} \chi\right) \square \phi-\left(m_{1}^{2}+m_{2}^{2}\right) \chi \nabla^{\mu} \phi \\
& -(\chi \leftrightarrow \phi) .
\end{aligned}
$$

If $\chi$ and $\phi$ are two solutions of the equation of motion (4.1), then one deduces that

$$
\nabla_{\mu} J^{\mu}[\chi, \phi]=0,
$$

and hence that the (pre)symplectic form $\omega(\chi, \phi)$ on the space of solutions of (4.1), given by

$$
\omega(\chi, \phi)=\int_{\Sigma} J^{\mu}[\chi, \phi] d \Sigma_{\mu}=-\omega(\phi, \chi),
$$

where $\Sigma$ is a Cauchy surface, is conserved.

Now we suppose that $\chi=\phi_{1}$ satisfies $\square \phi_{1}=\mu_{1}^{2} \phi_{1}$ and $\phi=\phi_{2}$ satisfies $\square \phi_{2}=\mu_{2}^{2} \phi_{2}$, where each of $\mu_{1}^{2}$ and $\mu_{2}^{2}$ can be either $m_{1}^{2}$ or $m_{2}^{2}$. Then

$J^{\mu}=\left(\mu_{1}^{2}+\mu_{2}^{2}-m_{1}^{2}-m_{2}^{2}\right)\left(\phi_{1} \nabla^{\mu} \phi_{2}-\phi_{2} \nabla^{\mu} \phi_{1}\right)$.

Thus the space of Cauchy data and the symplectic form splits as a direct sum of two summands, labeled by the mass-squared $m_{1}^{2}$ and $m_{2}^{2}$.

\section{Auxiliary field formulation}

A convenient way to handle the higher derivative terms in the Pais-Uhlenbeck equation is by introducing auxiliary fields. In particular, as we shall see below, this provides a simple way to calculate the contributions to the energymomentum tensor from the higher derivative terms. We shall employ two related, but slightly different, approaches in this paper. For now, we shall handle the various higher derivative terms that arise in a general Pais-Uhlenbeck theory separately, by developing an auxiliary-field construction for a Lagrangian $\mathcal{L}_{n}=-\frac{1}{2} \sqrt{-g} \phi(-\square)^{n} \phi$. The general Pais-Uhlenbeck (PU) Lagrangian is then given as a linear combination of such terms with certain constant coefficients. The energy-momentum tensor for the PU theory is then similarly given by the analogous linear combination of the associated energy-momentum tensors. In Appendix, we discuss a slightly different approach, in which the Lagrangian for the full PU field theory is directly rewritten by introducing auxiliary fields.

In the present simplest example we are considering, for which the PU action is given by (4.2), there is just the one higher derivative term, namely

$$
S_{(2)}[\phi]=-\frac{1}{2} \int_{D} \sqrt{-g} d^{4} x(\square \phi)^{2} .
$$

We now introduce an auxiliary field $\psi$ and replace (4.12) by

$$
\tilde{S}_{(2)}[\phi, \psi]=\int_{D} \sqrt{-g} d^{4} x\left(-\psi \square \phi+\frac{1}{2} \psi^{2}\right) .
$$

The variation of (4.13) with respect to $\psi$ yields

$$
\psi=\square \phi,
$$

and upon substituting this back into (4.13) one indeed recovers (4.12). The variation of (4.13) with respect to $\phi$ gives

$$
\square \psi=0,
$$

whence we obtain the field equation $\square^{2} \phi=0$ for $\phi$, which is indeed the same as the equation that arises directly from (4.12).

\section{Energy-momentum tensor}

Up to an integration by parts the action (4.13) is equivalent to

$\tilde{S}_{(2)}\left[\psi, \phi, g^{\mu \nu}\right]=\int_{D} \sqrt{-g} d^{4} x\left(\partial_{\mu} \psi g^{\mu \nu} \partial_{\nu} \phi+\frac{1}{2} \psi^{2}\right)$.

Now the associated Belinfante-Rosenfeld energymomentum tensor $T_{\mu \nu}^{(2)}$ is given by 


$$
\begin{aligned}
T_{\mu \nu}^{(2)} & =-2 \frac{\delta}{\delta g^{\mu \nu}} \tilde{S}_{2}\left[\psi, \phi, g^{\mu \nu}\right] \\
& =-\partial_{\mu} \psi \partial_{\nu} \phi-\partial_{\nu} \psi \partial_{\mu} \phi+g_{\mu \nu} \partial_{\alpha} \psi g^{\alpha \beta} \partial_{\beta} \phi+\frac{1}{2} g_{\mu \nu} \psi^{2} .
\end{aligned}
$$

Substituting (4.14) in (4.18) therefore gives

$$
\begin{aligned}
T_{\mu \nu}^{(2)}= & -\partial_{\mu} \square \phi \partial_{\nu} \phi-\partial_{\nu} \square \phi \partial_{\mu} \phi+g_{\mu \nu} \partial_{\alpha} \square \phi \partial^{\alpha} \phi \\
& +\frac{1}{2} g_{\mu \nu}(\square \phi)^{2},
\end{aligned}
$$

as the contribution to the energy-momentum tensor of the Pais-Uhlenbeck theory from the $(\square \phi)^{2}$ term in (4.2). One may verify that

$$
\nabla^{\mu} T_{\mu \nu}^{(2)}=-\square^{2} \phi \partial_{\nu} \phi,
$$

and so indeed this contribution to $T_{\mu \nu}$ is covariantly conserved if the field equation $\square^{2} \phi=0$ holds.

Combining the contribution $T_{\mu \nu}^{(2)}$ with the contributions from the remaining terms in (4.2), which are straightforward to calculate in the standard way, we find that the total energy-momentum tensor for this PU theory is given by

$$
\begin{aligned}
T_{\mu \nu}= & -\partial_{\mu} \square \phi \partial_{\nu} \phi-\partial_{\nu} \square \phi \partial_{\mu} \phi+g_{\mu \nu} \partial_{\alpha} \square \phi \partial^{\alpha} \phi \\
& +\frac{1}{2} g_{\mu \nu}(\square \phi)^{2}+\left(m_{1}^{2}+m_{2}^{2}\right) \\
& \left(\partial_{\mu} \phi \partial_{\nu} \phi-\frac{1}{2} g_{\mu \nu} g^{\alpha \beta} \partial_{\alpha} \phi \partial_{\beta} \phi\right) \\
& -\frac{1}{2} g_{\mu \nu} m_{1}^{2} m_{2}^{2} \phi^{2} .
\end{aligned}
$$

However, it should be noted that $T_{\mu \nu}$ is different, in its flat-spacetime limit, from the expression for $\bar{T}_{\mu \nu}$ given by (3.12). In Appendix we discuss a general derivation of the canonical energy-momentum tensor derived by the Noether method in flat spacetime and its relation to the energymomentum tensor that we obtained in (4.21) by using the Belinfante-Rosenfeld procedure.

Interestingly, although the flat space limit of (4.21) is not the same as (3.12), it gives the same expression (3.14) if one specializes to $\phi=\phi(t)$.

\section{B. Auxiliary fields}

\section{Powers of Laplace/d'Alembert operator}

Consider now the $n$th power of the d'Alembert operator $\square=\nabla^{2}$. By introducing auxiliary fields $\chi_{k}$ and Lagrange multipliers $\eta_{k}$, for $k=1, \ldots, n-1$, we can rewrite

$$
\begin{gathered}
\phi \square{ }^{n} \phi \rightarrow \phi \square \chi_{1}+\eta_{1}\left(\square \chi_{2}-\chi_{1}\right)+\eta_{2}\left(\square \chi_{3}-\chi_{2}\right)+\cdots \\
\quad+\eta_{n-2}\left(\square \chi_{n-1}-\chi_{n-2}\right)+\eta_{n-1}\left(\square \phi-\chi_{n-1}\right) .
\end{gathered}
$$

Indeed, varying with $\eta_{k}$ we find

$$
\begin{aligned}
\chi_{1} & =\square \chi_{2}, \quad \chi_{2}=\square \chi_{3}, \\
\cdots \chi_{n-1} & =\square \phi \Rightarrow \chi_{1}=\square^{n-1} \phi .
\end{aligned}
$$

On the other hand, varying with respect to $\chi_{k}$ we find that $\eta_{1}=\square \phi, \quad \eta_{2}=\square \eta_{1}, \quad \cdots \eta_{n-1}=\square^{n-2} \phi$.

This shows that we may identify

$$
\eta_{k}=\chi_{n-k}, \quad k=1, \ldots, n-1 .
$$

This identification reduces the number of extra fields by 2 . We have, for instance,

$$
\begin{aligned}
& \phi \square^{2} \phi=\phi \square \chi+\chi \square \phi-\chi^{2}, \\
& \phi \square^{3} \phi=\phi \square \chi_{1}+\chi_{2} \square \chi_{2}+\chi_{1} \square \phi-2 \chi_{2} \chi_{1} .
\end{aligned}
$$

If we also define $\chi_{0} \equiv 0$ and $\chi_{n} \equiv \phi$, then after making the identifications (4.25) we see that

$$
\phi \square \square^{n} \phi=\sum_{k=0}^{n-1} \chi_{n-k}\left(\square \chi_{k+1}-\chi_{k}\right) .
$$

Note that combined with the solutions (4.25) for the auxiliary fields, we have

$$
\chi_{k}=\square^{n-k} \chi, \quad 1 \leq k \leq n, \quad \chi_{0}=0 .
$$

Consider the Lagrangian

$$
\mathcal{L}=\sqrt{-g}\left[-\frac{1}{2} \phi(-\square)^{n} \phi-V\right]
$$

whose equations of motion are

$$
(-\square)^{n} \phi=-\frac{\partial V}{\partial \phi} .
$$

As established above, this can be written, after integrations by parts, as

$$
\mathcal{L}=\frac{1}{2}(-1)^{n} \sqrt{-g} \sum_{k=0}^{n-1}\left[\partial_{\mu} \chi_{n-k} \partial^{\mu} \chi_{k+1}+\chi_{n-k} \chi_{k}\right]-\sqrt{-g} V
$$

with the auxiliary fields given by

$$
\chi_{k}=\square^{n-k} \phi, \quad 1 \leq k \leq n, \quad \chi_{0}=0 .
$$

Calculating the energy-momentum tensor $T_{\mu \nu}=$ $-(2 / \sqrt{-g}) \delta \mathcal{L} / \delta g^{\mu \nu}$, we thus find 


$$
\begin{aligned}
T_{\mu \nu}= & (-1)^{n} \sum_{k=0}^{n-1}\left[-\partial_{\mu} \chi_{n-k} \partial_{\nu} \chi_{k+1}+\frac{1}{2} g_{\mu \nu} \partial_{\rho} \chi_{n-k} \partial^{\rho} \chi_{k+1}\right] \\
& +\frac{1}{2}(-1)^{n} g_{\mu \nu} \sum_{k=1}^{n-1} \chi_{n-k} \chi_{k}-g_{\mu \nu} V
\end{aligned}
$$

and hence, using (4.32),

$$
\begin{aligned}
T_{\mu \nu}= & (-1)^{n} \sum_{k=0}^{n-1}\left[-\partial_{\mu} \square^{k} \phi \partial_{\nu} \square^{n-k-1} \phi\right. \\
& \left.+\frac{1}{2} g_{\mu \nu} \partial_{\rho} \square^{k} \phi \partial^{\rho} \square^{n-k-1} \phi\right] \\
& +\frac{1}{2}(-1)^{n} g_{\mu \nu} \sum_{k=1}^{n-1} \square^{k} \phi \square^{n-k} \phi-g_{\mu \nu} V .
\end{aligned}
$$

(Note that the first term is, in fact, automatically symmetric in $\mu$ and $\nu$, in view of the summation over $k$.) The first few examples, for $n=1,2$, and 3 , are

$$
\begin{aligned}
n=1: T_{\mu \nu}= & \partial_{\mu} \phi \partial_{\nu} \phi-\frac{1}{2} g_{\mu \nu} \partial_{\rho} \phi \partial^{\rho} \phi-g_{\mu \nu} V, \\
n=2: T_{\mu \nu}= & -\partial_{\mu} \phi \partial_{\nu} \square \phi-\partial_{\nu} \phi \partial_{\mu} \square \phi+g_{\mu \nu} \partial_{\rho} \square \phi \partial^{\rho} \phi \\
& +\frac{1}{2} g_{\mu \nu}(\square \phi)^{2}-g_{\mu \nu} V, \\
n=3: T_{\mu \nu}= & \partial_{\mu} \phi \partial_{\nu} \square^{2} \phi+\partial_{\mu} \square \phi \partial_{\nu} \square \phi+\partial_{\mu} \square^{2} \phi \partial_{\nu} \phi \\
& -g_{\mu \nu} \partial_{\rho} \square^{2} \phi \partial^{\rho} \phi-\frac{1}{2} g_{\mu \nu} \partial_{\rho} \square \phi \partial^{\rho} \square \phi \\
& -g_{\mu \nu} \square \phi \square^{2} \phi-g_{\mu \nu} V .
\end{aligned}
$$

\section{Auxiliary fields for general higher-order operators}

Consider the higher derivative Lagrangian

$$
L=\phi A_{1} A_{2} \cdots A_{n} \phi
$$

where the operators $A_{i}$ are formally self-adjoint and need not necessarily be mutually commuting. An example for which they are mutually commuting is

$$
A_{i}=\square-m_{i}^{2}, \quad \square=\nabla^{2} .
$$

Paralleling the procedure for introducing auxiliary fields for the Lagrangian $L_{n}=\phi \Delta^{n} \phi$, consider here

$$
\begin{aligned}
L= & \phi A_{1} \chi_{1}+\eta_{1}\left(A_{2} \chi_{2}-\chi_{1}\right)+\eta_{2}\left(A_{3} \chi_{3}-\chi_{2}\right)+\cdots \\
& +\eta_{n-2}\left(A_{n-1} \chi_{n-1}-\chi_{n-2}\right)+\eta_{n-1}\left(A_{n} \phi-\chi_{n-1}\right) .
\end{aligned}
$$

Varying the $\eta_{i}$ fields gives

$$
\begin{aligned}
\chi_{1} & =A_{2} \chi_{2}, \quad \chi_{2}=A_{3} \chi_{3}, \quad \ldots, \quad \chi_{n-1}=A_{n} \phi \\
& \Rightarrow \chi_{1}=A_{2} A_{3} \cdots A_{n} \phi .
\end{aligned}
$$

Varying the $\chi_{i}$ fields gives

$\eta_{1}=A_{1} \phi, \quad \eta_{2}=A_{2} \eta_{1}, \quad \ldots, \quad \eta_{n-1}=A_{n-1} \eta_{n-2}$.

Unlike in the case of the pure $\square^{n}$ Lagrangian, we cannot equate the set of $\eta_{i}$ fields with the set of $\chi_{i}$ fields.

It is easily seen that the Lagrangian (4.38) gives rise to (4.36) after plugging in the solutions for the $\chi_{i}$ fields, given in (4.39). Note that if we define

$$
\chi_{0} \equiv 0, \quad \chi_{n} \equiv \phi, \quad \eta_{0} \equiv \phi, \quad \eta_{n} \equiv 0,
$$

then the Lagrangian (4.38) can be written as

$$
L=\sum_{k=0}^{n-1} \eta_{k}\left(A_{k+1} \chi_{k+1}-\chi_{k}\right) .
$$

Note that together with the solutions (4.39) and (4.40) for the auxiliary fields, we have ${ }^{5}$

$$
\begin{aligned}
\chi_{k} & =A_{k+1} A_{k+2} \cdots A_{n} \phi, & 1 \leq k \leq n, & \chi_{0}=0, \\
\eta_{k} & =A_{k} A_{k-1} \cdots A_{1} \phi, & 0 \leq k \leq n-1, & \eta_{n}=0 .
\end{aligned}
$$

If we consider the example where $A_{i}$ is given by (4.37), the energy-momentum tensor that follows from (4.42) is

$$
\begin{aligned}
T_{\mu \nu}= & \sum_{k=0}^{n-1}\left[\partial_{(\mu} \eta_{k} \partial_{\nu)} \chi_{k+1}-\frac{1}{2} g_{\mu \nu}\left(\partial_{\rho} \eta_{k} \partial^{\rho} \chi_{k+1}\right.\right. \\
& \left.\left.+m_{k+1}^{2} \eta_{k} \chi_{k+1}+\eta_{k} \chi_{k}\right)\right] .
\end{aligned}
$$

\section{Symplectic current in higher derivative scalar theory}

Consider first the Lagrangian $\frac{1}{2} \phi \square^{n} \phi$, whose equation of motion is

\footnotetext{
${ }^{5}$ The discussion we gave in Sec. IV B, where we introduced auxiliary fields for the Lagrangian $\phi \square^{n} \phi$, is a special case of our discussion here, in which $A_{k}=\square$ for all $k$. As can be seen from (4.39) and (4.40), in this special case one can equate $\eta_{k}=\chi_{n-k}$ as in Sec. IV B. But in the more general case discussed in this section, one cannot equate the $\eta$ and $\chi$ fields, even in the case that the operators $A_{i}$ commute. If, however, the operators $A_{i}$ take the form given in (4.37), where the differential operator parts of all the $A_{i}$ are the same, then there exist purely algebraic relations between the set of $\eta_{k}$ fields and the set of $\chi_{k}$ fields, as can be seen from (4.43). In this case, therefore, one needs only $\phi$ and either the set of $\chi_{k}$ or the set of $\eta_{k}$ as auxiliary fields.
} 


$$
\square^{n} \phi=0 .
$$

By extracting derivatives in sequence from

$$
\psi \square^{n} \phi-\phi \square^{n} \psi,
$$

we obtain

$$
\begin{aligned}
\psi & \square^{n} \phi-\phi \square^{n} \psi \\
= & \nabla_{\mu}\left(\psi \nabla^{\mu} \square^{n-1} \phi\right)-\nabla_{\mu} \psi \nabla^{\mu} \square^{n-1} \phi-(\psi \leftrightarrow \phi) \\
= & \nabla_{\mu}\left(\psi \nabla^{\mu} \square^{n-1} \phi-\nabla^{\mu} \psi \square^{n-1} \phi\right)+\square \psi \square^{n-1} \phi \\
& \quad-(\psi \leftrightarrow \phi) \\
= & \cdots \\
= & \sum_{p=0}^{n-1} J^{\mu}\left(\square^{p} \psi, \square^{n-p-1} \psi\right),
\end{aligned}
$$

where we have defined

$$
J^{\mu}(A, B) \equiv A \nabla^{\mu} B-B \nabla^{\mu} A .
$$

Thus, if $\phi$ and $\psi$ are any solutions of $\square^{n} \phi=0$ and $\square^{n} \psi=0$, we have a conserved current

$$
J_{\square^{n}}^{\mu}(\psi, \phi)=\sum_{p=0}^{n-1} J^{\mu}\left(\square^{p} \psi, \square^{n-p-1} \phi\right) .
$$

Consider now the Lagrangian, and consequent equations of motion,

$$
\begin{aligned}
\mathcal{L} & =\frac{1}{2} \phi \prod_{i=1}^{N}\left(\square-m_{i}^{2}\right) \phi, \\
\Delta_{N} \phi & \equiv \prod_{i=1}^{N}\left(\square-m_{i}^{2}\right) \phi=0 .
\end{aligned}
$$

If we assume the masses are all unequal, the general solution is a linear combination of "elementary solutions" satisfying

$$
\left(\square-m_{i}^{2}\right) \phi_{i}=0,
$$

for any $i$ in the range $1 \leq i \leq N$.

We now consider the conserved current $J_{\Delta_{N}}^{\mu}$ for the operator $\Delta_{N}$ defined in (4.50). If we write the operator as

$$
\Delta_{N}=\sum_{n=0}^{N} a_{n} \square^{n}
$$

then this current is given by

$$
J_{\Delta_{N}}^{\mu}(\psi, \phi)=\sum_{n=1}^{N} a_{n} J_{\square^{n}}^{\mu}(\psi, \phi),
$$

where $J_{\square^{n}}^{\mu}(\psi, \phi)$ is defined in (4.49).

There are two cases of particular interest to consider. The first is when $\psi$ and $\phi$ are two "elementary" solutions of $\Delta_{N}$, as defined in (4.51), corresponding to two different $m_{i}^{2}$ values. Without loss of generality we may take $\psi=\phi_{1}$ and $\phi=\phi_{2}$; that is to say they satisfy

$$
\square \psi=m_{1}^{2} \psi, \quad \square \phi=m_{2}^{2} \phi .
$$

Writing the operator $\Delta_{N}$ as

$$
\Delta_{N}=\left(\square-m_{1}^{2}\right)\left(\square-m_{2}^{2}\right) f(\square),
$$

with

$$
f(\square)=\prod_{i=3}^{N}\left(\square-m_{i}^{2}\right)=\sum_{k=0}^{N-2} b_{k} \square \square^{k},
$$

and noting that

$$
J^{\mu}\left(\square^{p} \psi, \square^{(n-p-1)} \phi\right)=m_{1}^{2 p} m_{2}^{2 n-2 p-2} J_{\square}^{\mu}(\psi, \phi),
$$

we see that

$$
\begin{aligned}
J_{\Delta_{N}}^{\mu}(\psi, \phi)= & \sum_{k=0}^{N-2} b_{k}\left[\sum_{p=0}^{k+1} m_{1}^{2 p} m_{2}^{2 k-2 p+2}-\left(m_{1}^{2}+m_{2}^{2}\right)\right. \\
& \left.\times \sum_{p=0}^{k} m_{1}^{2 p} m_{2}^{2 k-2 p}+m_{1}^{2} m_{2}^{2} \sum_{p=0}^{k-1} m_{1}^{2 p} m_{2}^{2 k-2 p-2}\right] \\
& \times J_{\square}^{\mu}(\psi, \phi) .
\end{aligned}
$$

Collecting the terms in the square brackets as a sum $\sum_{p=0}^{k-1}$ together with the additional terms from the first two summations, we immediately find that the total vanishes for each $k$. Thus we find

$$
J_{\Delta_{N}}^{\mu}(\psi, \phi)=0,
$$

whenever $\psi$ and $\phi$ are elementary solutions with different mass values.

The other case of interest is when $\psi$ and $\phi$ are solutions with the same mass value. Without loss of generality, we may consider the case where $\psi$ and $\phi$ satisfy

$$
\square \psi=m_{1}^{2} \psi, \quad \square \phi=m_{1}^{2} \phi .
$$

This means that $J_{\square^{n}}^{\mu}(\psi, \phi)=m_{1}^{2 n-2} J_{\square}^{\mu}(\psi, \phi)$. Writing the operator $\Delta_{N}$ as 


$$
\Delta_{N}=\left(\square-m_{1}^{2}\right) h(\square)
$$

with

$$
h(\square)=\prod_{i=2}^{N}\left(\square-m_{i}^{2}\right)=\sum_{k=0}^{N-1} c_{k} \square^{k},
$$

we find that

$$
\begin{aligned}
J_{\Delta_{N}}^{\mu}(\psi, \phi) & =\sum_{k=0}^{N-1} c_{k}\left[J_{\square^{k+1}}^{\mu}(\psi, \phi)-m_{1}^{2} J_{\square^{k}}^{\mu}(\psi, \phi)\right] \\
& =\sum_{k=0}^{N-1} c_{k}\left[(k+1) m_{1}^{2 k}-k m_{1}^{2 k}\right] J_{\square}^{\mu}(\psi, \phi) \\
& =\sum_{k=0}^{N-1} c_{k} m_{1}^{2 k} J_{\square}^{\mu}(\psi, \phi) \\
& =h\left(m_{1}^{2}\right) J_{\square}^{\mu}(\psi, \phi) \\
& =\prod_{i=2}^{N}\left(m_{1}^{2}-m_{i}^{2}\right) J_{\square}^{\mu}(\psi, \phi) .
\end{aligned}
$$

In other words, when $\psi$ and $\phi$ are solutions with the same mass, as in (4.60), the conserved current is given by

$$
J_{\Delta_{N}}^{\mu}(\psi, \phi)=\prod_{i=2}^{N}\left(m_{1}^{2}-m_{i}^{2}\right) J^{\mu}(\psi, \phi),
$$

where $J^{\mu}(\psi, \phi)$ is the usual current

$$
J^{\mu}(\psi, \phi)=\psi \nabla^{\mu} \phi-\phi \nabla^{\mu} \psi .
$$

In the general case when $\square \psi=y^{2} \psi$ and $\square \phi=x^{2} \phi$, we have

$$
\Delta_{N}=\prod_{i=1}^{N}\left(\square-m_{i}^{2}\right)=W(\square)=\sum_{k=0}^{N} a_{k} \square^{k}
$$

and

$$
J_{\Delta_{N}}^{\mu}(\psi, \phi)=\sum_{k=1}^{N} a_{k} J_{\square^{k}}^{\mu}(\psi, \phi) .
$$

From (4.49) we therefore have

$$
\begin{aligned}
J_{\Delta_{N}}^{\mu}(\psi, \phi) & =\sum_{k=1}^{N} a_{k} x^{2 k-2} \sum_{p=0}^{k-1}\left(\frac{y}{x}\right)^{2 p} J^{\mu}(\psi, \phi) \\
& =\sum_{k=1}^{N} a_{k} x^{2 k-2}\left(\frac{1-\left(\frac{y}{x}\right)^{2 k}}{1-\left(\frac{y}{x}\right)^{2}}\right) J^{\mu}(\psi, \phi) \\
& =\frac{1}{x^{2}-y^{2}} \sum_{k=1}^{N} a_{k}\left(x^{2 k}-y^{2 k}\right) J^{\mu}(\psi, \phi) \\
& =\frac{1}{x^{2}-y^{2}} \sum_{k=0}^{N} a_{k}\left(x^{2 k}-y^{2 k}\right) J^{\mu}(\psi, \phi) \\
& =\frac{1}{x^{2}-y^{2}}\left(W\left(x^{2}\right)-W\left(y^{2}\right)\right) J^{\mu}(\psi, \phi),
\end{aligned}
$$

and hence we have

$J_{\Delta_{N}}^{\mu}(\psi, \phi)=\frac{\prod_{i=1}^{N}\left(y^{2}-m_{i}^{2}\right)-\prod_{i=1}^{N}\left(x^{2}-m_{i}^{2}\right)}{y^{2}-x^{2}} J^{\mu}(\psi, \phi)$.

\section{GREEN FUNCTIONS AND EUCLIDEAN FORMULATION}

Pais and Uhlenbeck solved for the time-independent Green function $G^{(4)}(\mathbf{r})$ for the fourth-order operator in flat space, defined by

$$
\left(-\nabla^{2}+m_{1}^{2}\right)\left(-\nabla^{2}+m_{2}^{2}\right) G^{(4)}(\mathbf{r})=\delta(\mathbf{r}),
$$

where $\mathbf{r}=\mathbf{r}_{1}-\mathbf{r}_{2}$, by using Fourier transforms. Since

$$
\begin{aligned}
& \frac{1}{\left(-\nabla^{2}+m_{1}^{2}\right)\left(-\nabla^{2}+m_{2}^{2}\right)} \\
& \quad=\frac{1}{m_{2}^{2}-m_{1}^{2}}\left[\frac{1}{\left(-\nabla^{2}+m_{1}^{2}\right)}-\frac{1}{\left(-\nabla^{2}+m_{2}^{2}\right)}\right],
\end{aligned}
$$

the formal solution ${ }^{6}$ is

$$
G^{(4)}(\mathbf{r})=\frac{\left(e^{-m_{1} r}-e^{-m_{2} r}\right)}{4 \pi\left(m_{2}^{2}-m_{1}^{2}\right) r},
$$

where $r=|\mathbf{r}| \cdot G^{(4)}(\mathbf{r})$ goes to a constant value at the origin, and it admits a Taylor expansion in powers of $r$. However, because the expansion includes odd powers of $r$, it is not differentiable with respect to the Cartesian coordinates at the origin $r=0$, and indeed for this reason it does, in fact, satisfy (5.1) with the delta function on the right-hand side. This may easily be verified by integrating (5.1) over a small spherical volume centered on the origin, and using the divergence theorem to turn the derivative terms into surface

\footnotetext{
${ }^{6}$ Pais and Uhlenbeck omit the denominator $\left(m_{2}^{2}-m_{1}^{2}\right)$.
} 
integrals. Note that $G^{(4)}(\mathbf{r})$ is symmetric under the interchange of $m_{1}$ and $m_{2}$. Both $\left(-\nabla^{2}+m_{1}^{2}\right)$ and $\left(-\nabla^{2}+m_{2}^{2}\right)$ are positive operators, and their Green functions $\frac{e^{-m_{1} r}}{4 \pi r}$ and $\frac{e^{-m_{2} r}}{4 \pi r}$ are both positive. The operator $\left(-\nabla^{2}+m_{1}^{2}\right)\left(-\nabla^{2}+\right.$ $\left.m_{2}^{2}\right)$ is positive and, as one may check, $G^{(4)}(\mathbf{r})$ is also positive.

The Green function (5.3) is unique, since there are no nonsingular solutions of the homogeneous equation

$$
\nabla^{4} \phi-\left(m_{1}^{2}+m_{2}^{2}\right) \nabla^{2} \phi+m_{1}^{2} m_{1}^{2} \phi=0
$$

that fall off sufficiently fast at infinity. This is easily established by multiplying (5.4) by $\phi$ and integrating over $E^{3}$. Integrating by parts gives

$$
\int_{E^{3}}\left[\left(\nabla^{2} \phi\right)^{2}+\left(m_{1}^{2}+m_{2}^{2}\right)(\nabla \phi)^{2}+m_{1}^{2} m_{2}^{2} \phi^{2}\right] d^{3} x=0,
$$

whence $\phi=0$.

Similar conclusions hold in four-dimensional Euclidean space, but the explicit form of the Green function is more complicated since it involves Bessel functions. Concretely, the Euclidean Green function $G_{E}(r)$ for the second-order Klein-Gordon operator, obeying $\left(-\nabla^{2}+m^{2}\right) G_{E}(r)=\delta(\mathbf{r})$, is given by

$$
G_{E}(r)=\frac{m K_{1}(m r)}{4 \pi^{2} r}
$$

where $K_{\nu}(x)$ is the modified Bessel function of the second kind, which has the integral representation

$$
K_{\nu}(x)=\frac{\sqrt{\pi} x^{\nu}}{2^{\nu} \Gamma\left(\nu+\frac{1}{2}\right)} \int_{1}^{\infty} d t e^{-x t}\left(t^{2}-1\right)^{\nu-\frac{1}{2}} .
$$

At small $r$ the Green function $G_{E}(\mathbf{r})$ has the expansion

$$
G_{E}(\mathbf{r})=\frac{1}{4 \pi^{2} r^{2}}+\frac{m^{2}}{16 \pi^{2}}\left(2 \gamma+2 \log \frac{m r}{2}-1\right)+\cdots,
$$

where $\gamma$ is the Euler-Mascheroni constant. For the fourthorder Pais-Uhlenbeck operator, the corresponding Green function $G_{E}^{(4)}(r)$ obeys (5.1) (with $\nabla^{2}$ now understood to be the four-dimensional Euclidean Laplacian) and is given by

$$
G_{E}^{(4)}(\mathbf{r})=\frac{m_{1} K_{1}\left(m_{1} r\right)-m_{2} K_{1}\left(m_{2} r\right)}{4 \pi^{2}\left(m_{2}^{2}-m_{1}^{2}\right) r} .
$$

One may easily verify, for example by using (5.7), that both (5.8) and (5.9) are positive. [Note that the $r^{-2}$ singularity in (5.8) is canceled in (5.9).] Because of the positivity of $G_{E}^{(4)}(\mathbf{r})$, one might be led to believe that the OsterwalderSchrader construction of the quantum field theory could be carried out with no negative norm states. This, however, is not the case [15], as we shall discuss below.

\section{A. Reflection positivity in the fourth-order case}

If we work on a Riemannian manifold, the Euclidean action functional

$$
\begin{aligned}
I= & \frac{1}{2} \int_{D} \sqrt{g} d^{4} x\left[\left(\nabla^{2} \phi\right)^{2}+\left(m_{1}^{2}+m_{2}^{2}\right)\left(g^{\mu \nu} \partial_{\mu} \phi \partial_{\nu} \phi\right)\right. \\
& \left.+m_{1}^{2} m_{2}^{2} \phi^{2}\right]
\end{aligned}
$$

is positive definite. Thus the Euclidean functional integrals should make sense and the associated two-point function or Green function

$$
G_{E}=\frac{1}{m_{2}^{2}-m_{1}^{2}}\left[\frac{1}{\left(-\nabla^{2}+m_{1}^{2}\right)}-\frac{1}{\left(-\nabla^{2}+m_{2}^{2}\right)}\right],
$$

being the inverse of the positive operator, will be positive.

In general, given a Riemannian manifold there is no Lorentzian manifold associated with it by analytic continuation. If there is, one may analytically continue the two-point function and ask what, if any, is its physical significance. In the case of Euclidean space $\mathbb{E}^{4}$ it is well known that one obtains the Wightman function associated with the vacuum state. If one periodically identifies one of the coordinates with period $\beta$, one obtains expectation values in the Gibbs state at temperature $T=\frac{1}{\beta}$. In fact, using a construction due to Osterwalder and Schrader one need not depart from Euclidean space in order to construct the quantum mechanical Hilbert space. This will be possible as long as the Green function satisfies the requirement of reflection positivity [20]. ${ }^{7}$ It has been suggested by a number of authors [28-36] that this construction may be generalized to a restricted class of Riemannian manifolds, including globally static spacetimes, which more generally may be characterized as "real tunneling geometries" [19].

One requires that the Riemann manifold admit a reflection symmetry $\theta$, an involutive isometry which leaves fixed a two-sided hypersurface $\Sigma$ such that

$$
M=M_{-} \sqcup \Sigma \sqcup M_{+}, \quad \theta M_{ \pm}=M_{\mp},
$$

where $\sqcup$ denotes the disjoint union. Let $V$ be the space of complex valued functions that vanish outside $M_{+}$. If $|f\rangle \in V$ and $\bar{f}_{\theta}^{\star}=\bar{f}(\theta(x))$ is the pullback of its complex conjugate under $\theta$, we define

$$
\begin{aligned}
\langle f \mid f\rangle & =\| f\rangle\left.\right|^{2} \\
& =\iint d^{4} x \sqrt{g(x)} d^{4} y \sqrt{g(y)} \bar{f}(\theta x) G(x, y) f(y) .
\end{aligned}
$$

\footnotetext{
${ }^{7}$ For a helpful introduction the reader may consult [27].
} 
If the right-hand side of (5.13) is positive definite, then the left-hand side provides a Hermitian inner product on $V$ which may be identified with the one-particle Hilbert space.

If

$$
G_{E}=\frac{1}{\left(-\nabla^{2}+m^{2}\right)},
$$

then this reflection positivity condition is satisfied [35]. However, if

$$
\begin{aligned}
G_{E}^{(4)} & =\frac{1}{\left(-\nabla^{2}+m_{2}^{2}\right)\left(-\nabla^{2}+m_{1}^{2}\right)} \\
& =\frac{1}{m_{1}^{2}-m_{2}^{2}}\left[\frac{1}{\left(-\nabla^{2}+m_{2}^{2}\right)}-\frac{1}{\left(-\nabla^{2}+m_{1}^{2}\right)}\right],
\end{aligned}
$$

then reflection positivity is not satisfied [15]. To see this, suppose $m_{1}^{2}>m_{2}^{2}$ and let

$$
f=\left(-\nabla^{2}+m_{2}^{2}\right) h
$$

where $h \in V$. Then

$$
\begin{aligned}
\langle f \mid f\rangle= & \frac{1}{m_{1}^{2}-m_{2}^{2}} \int \sqrt{g(x)} d^{4} x \int \sqrt{g(y)} d^{4} y \\
& \times\left[\bar{f}(\theta x) h-\bar{f}(\theta x) \frac{1}{\left(-\nabla^{2}+m_{1}^{2}\right)} f(y)\right] \\
= & -\frac{1}{m_{1}^{2}-m_{2}^{2}} \int \sqrt{g(x)} d^{4} x \int \sqrt{g(y)} d^{4} y \bar{f}(\theta x) \\
& \times \frac{1}{\left(-\nabla^{2}+m_{1}^{2}\right)} f(y),
\end{aligned}
$$

since $\bar{f}(\theta x)$ and $h$ have disjoint support. Thus states of the form $\left|\left(-\nabla^{2}+m_{2}^{2}\right) h\right\rangle$ have negative norm squared.

\section{B. 2Nth-order case}

For the $2 N$ th-order operator we have

$$
\prod_{i=1}^{N} \frac{1}{\left(-\nabla^{2}+m_{i}^{2}\right)}=\sum_{i=1}^{N} \frac{1}{C_{i}} \frac{1}{\left(-\nabla^{2}+m_{i}^{2}\right)},
$$

where

$$
C_{i}=\prod_{j \neq i}\left(m_{j}^{2}-m_{i}^{2}\right)
$$

Proceeding as before, and considering

$$
f_{i}=\prod_{j \neq i}\left(-\nabla^{2}+m_{j}^{2}\right) h
$$

we shall have

$$
\begin{aligned}
\left\langle f_{i} \mid f_{i}\right\rangle= & \frac{1}{C_{i}} \int \sqrt{g(x)} d^{4} x \int \sqrt{g(y)} d^{4} y \bar{f}_{i}(\theta x) \\
& \times \frac{1}{\left(-\nabla^{2}+m_{i}^{2}\right)} f_{i}(y) .
\end{aligned}
$$

This can have either sign, depending on the sign of $C_{i}$, and this depends upon the relative values for the masses $m_{i}$. For example, if we order the masses so that

$$
m_{1}^{2}>m_{2}^{2}>m_{3}^{2}>\ldots>m_{N}^{2},
$$

then $C_{N}$ is positive, and the signs of $C_{i}$ alternate as $i$ decreases. Thus there will always be states of negative norm as well as states of positive norm.

It is interesting to note that if we construct the static Green function $G^{(2 N)}(\mathbf{r})$ generalising (5.3) to the $2 N$ th-order operator, we obtain

$$
G^{(2 N)}(\mathbf{r})=\frac{1}{4 \pi r} \sum_{i=1}^{N} \frac{e^{-m_{i} r}}{C_{i}},
$$

and that this is finite at $r=0$ for all $N \geq 2$. In fact, one can see that $^{8}$

$$
\sum_{i=1}^{N} \frac{m_{i}^{2 p}}{C_{i}}=0, \quad \text { for } p \leq N-2
$$

and that

$$
\sum_{i=1}^{N} \frac{m_{i}^{2 N-2}}{C_{i}}=(-1)^{N-1}
$$

This implies that the Taylor expansion of $G^{(2 N)}(\mathbf{r})$ in (5.24) has the form

$$
\begin{aligned}
G^{(2 N)}(\mathbf{r})= & a_{0}+a_{2} r^{2}+a_{4} r^{4}+a_{6} r^{6}+\cdots \\
& +\frac{(-1)^{N-1}}{4 \pi(2 N-2) !} r^{2 N-3}+a_{2 N-1} r^{2 N-1} \\
& +a_{2 N+1} r^{2 N+1}+\cdots,
\end{aligned}
$$

where the constant $a_{k}$ coefficients depend on the masses $m_{i}$. The nonanalyticity of the Green function, as a function of the Cartesian coordinates, is associated with the occurrence of odd-integer powers of $r$ in the Taylor expansion (5.27).

\footnotetext{
${ }^{8}$ These results can be proved by considering the contour integrals

$$
\oint_{C} d z z^{p} \prod_{i=1}^{N} \frac{1}{\left(z-m_{i}^{2}\right)}
$$

for $0 \leq p \leq N-1$, where the contour $C$ is taken to be a circle of radius $R$ centered on the origin, in the limit where $R$ is sent to infinity.
} 
The first such term, for the $2 N$ th-order operator, occurs at the order $r^{2 N-3}$.

This softening of the Green function at short distance is analogous to the softening encountered in Pauli-Villars regularization [37].

The Euclidean Green function $G_{E}^{(2 N)}(\mathbf{r})$ for the $2 N$ thorder Pais-Uhlenbeck operator can also be calculated using the same formalism as above. From (5.6), we see that

$$
G_{E}^{(2 N)}(\mathbf{r})=\sum_{i=1}^{N} \frac{m_{i} K_{1}\left(m_{i} r\right)}{4 \pi^{2} C_{i} r} .
$$

The identities (5.25) and (5.26) again imply that the nonanalytic behavior of $G_{E}^{(2 N)}(\mathbf{r})$ [in the form of the $\log \left(m_{i} r / 2\right)$ terms] is deferred to an increasingly higher order in a small- $r$ expansion as $N$ increases.

\section{Higher dimensional green functions}

The Green function $G_{E}(\mathbf{r})$ in $d$-dimensional Euclidean space, obeying $\left(-\nabla^{2}+m^{2}\right) G_{E}(\mathbf{r})=\delta(\mathbf{r})$, is given by

$$
G_{E}(\mathbf{r})=\frac{m^{\frac{d}{2}-1} K_{\frac{d}{2}-1}(m r)}{(2 \pi)^{\frac{d}{2}} r^{\frac{d}{2}-1}} .
$$

The cases we discussed previously correspond to $d=3$, for the static Green function in four-dimensional spacetime, and $d=4$, for the four-dimensional Euclidean Green function. The analysis of the Euclidean Green functions for the higher-order Pais-Uhlenbeck operators proceeds in the same manner as in the cases we discussed previously. As in those cases, the onset of nonanalytic behavior in the Green functions for the higher-order Pais-Uhlenbeck operators, namely

$$
G_{E}^{(2 N)}(\mathbf{r})=\sum_{i=1}^{N} \frac{m_{i}^{\frac{d}{2}-1} K_{\frac{d}{2}-1}\left(m_{i} r\right)}{(2 \pi)^{\frac{d}{2}} C_{i} r^{\frac{d}{2}-1}},
$$

is deferred to increasingly higher powers in the small- $r$ expansion as $N$ increases.

\section{NO-HAIR THEOREMS FOR STATIC AND STATIONARY SOLUTIONS}

In this section, we address the question of whether there could exist regular static solutions to certain classes of higher derivative field theories. First, we consider field theories of the Pais-Uhlenbeck type. Then, we consider a class of $2 \mathrm{~N}$-order field theories with a general potential $V(\phi)$.

\section{A. Pais-Uhlenbeck field theories}

Our discussion here applies rather generally to the situation where we have a static asymptotically flat background metric, regular outside a regular event horizon, in which a scalar field that is also static satisfies the PaisUhlenbeck equations

$$
\prod_{i=1}^{N}\left(\square-m_{i}^{2}\right) \phi=0 .
$$

For concreteness, we shall first consider the fourth-order example (4.1). If we integrate over the domain $D$ obtained by taking an initial spacelike surface $\Sigma_{(i)}$ extending from infinity to the horizon, and moving it up the orbits of the static Killing vector field $K^{\mu}$ that coincides on the horizon with its null generators, to a final surface $\Sigma_{(f)}$, and then use (4.6) with $\chi=\phi$, we find that

$$
\begin{aligned}
& \frac{1}{2} \int\left(\left(\nabla^{2} \phi\right)^{2}+\left(m_{1}^{2}+m_{2}^{2}\right) g^{\mu \nu} \partial_{\mu} \phi \partial_{\nu} \phi+m_{1}^{2} m_{2}^{2} \phi^{2}\right) \sqrt{-g} d^{4} x \\
& =\int_{\partial D}\left(-\phi\left(\nabla^{\mu} \nabla^{2} \phi+\left(\nabla^{\mu} \phi\right)\left(\nabla^{2} \phi\right)+\left(m_{1}^{2}+m_{2}^{2}\right) \phi \nabla^{\mu} \phi\right) d \Sigma_{\mu} .\right.
\end{aligned}
$$

Now $\partial D$ has four boundary components: One is at infinity, $\partial D_{\infty}$; one is on the horizon, $\partial D_{H}$; one is the initial surface $\partial D_{i}=\Sigma_{(i)}$; and the fourth is the final surface $\partial D_{f}=\Sigma_{(f)}$. The integrals over $\partial D_{i}$ and $\partial D_{f}$ cancel one another, since, by construction, $\Sigma_{(f)}$ is obtained by carrying $\Sigma_{(i)}$ along the orbits of the Killing vector $K^{\mu}$. The integrand on $\partial D_{H}$ vanishes because $d \Sigma_{\mu}$ on $H$ is proportional to $K^{\mu}$ and $K^{\mu} \partial_{\mu} \phi=K^{\mu} \partial_{\mu} \nabla^{2} \phi=0$. The integral over $\partial D_{\infty}$ vanishes by the boundary conditions on $\phi$.

Thus the left-hand side of (6.2) vanishes. But, unless $m_{1}^{2} m_{2}^{2}=0$, the integrand is positive definite. Thus in this case $\phi=0$. If it happens that $m_{1}^{2} m_{2}^{2}=0$ but $m_{1}^{2}+m_{2}^{2} \neq 0$, we may only deduce that $\phi=$ const. If $m_{1}^{2}+m_{2}^{2}=0$, we deduce that $\nabla^{2} \phi=0$ and hence, by a standard argument, that $\phi=$ const in this case as well. Thus we have shown that there can exist no regular, static solutions for the scalar field $\phi$ in a static black hole background. A special case of this result is when there is no black hole, and the spacetime is just flat Minkowski spacetime.

The above argument generalizes immediately to the $2 N$-order Pais-Uhlenbeck theory (6.1), since by appropriate integrations by parts, the analogue of the left-hand side in (6.2) will consist of a sum of squares with positive coefficients. The analogue of the surface terms on the righthand side will again vanish, by virtue of the assumption of staticity and the imposition of an appropriate boundary condition on $\phi$ at infinity. Thus again, one concludes that there can be no nontrivial static solutions for $\phi$ in flat space or a regular static black hole background.

The result also immediately generalizes to higher spacetime dimensions. 
One may also consider adding a scalar potential $V(\phi)$. If this is such that $\phi V^{\prime}(\phi)$ is everywhere non-negative, then one will again be able to show that $\phi$ must vanish for a static solution.

\section{B. Higher-order theories with a general potential}

In this section, we suppose that $\phi$ is static and obeys

$$
-(-\square)^{n} \phi=V^{\prime}(\phi)
$$

in a curved $d$-dimensional spacetime. Differentiating with respect to $\partial_{\mu}$ and then contracting with $\partial^{\mu} \phi$ implies

$$
-\partial^{\mu} \phi \partial_{\mu}(-\square)^{n} \phi=V^{\prime \prime} g^{\mu \nu} \partial_{\mu} \phi \partial_{\nu} \phi .
$$

By extracting derivatives sequentially, this can be written in the form

$$
\nabla_{\mu} S_{(n)}^{\mu}-\left(\square \frac{1}{2}(n+1) \phi\right)^{2}=V^{\prime \prime} g^{\mu \nu} \partial_{\mu} \phi \partial_{\nu} \phi
$$

if $n$ is odd, or as

$$
\nabla_{\mu} S_{(n)}^{\mu}-\left(\nabla_{\mu} \square \frac{1}{2} n \phi\right)^{2}=V^{\prime \prime} g^{\mu \nu} \partial_{\mu} \phi \partial_{\nu} \phi
$$

if $n$ is even.

If we assume that the solution $\phi$ is static, and that the metric is static, then we can proceed as in Sec. VIA by integrating (6.5) or (6.6) over a portion of spacetime bounded by initial and final surfaces $\Sigma_{(i)}$ and $\Sigma_{(f)}$, a horizon $H$, and a boundary at infinity. For the same reasons as in Sec. VI A, the boundary contributions on $\Sigma_{(i)}$ and $\Sigma_{(f)}$ will cancel, the term on the horizon will vanish, and the contribution at infinity will give zero subject to appropriate falloff conditions for $\phi$. We may then conclude that if the potential is convex, i.e., $V^{\prime \prime} \geq 0$ everywhere, then $\phi$ must be a constant.

By a straightforward extension of the argument above, we can similarly conclude that there are no nontrivial static solutions to the equation

$$
(-1)^{N+1} F(\square) \phi=V^{\prime}(\phi),
$$

where

$$
F(\square)=\prod_{i=1}^{N}\left(\square-m_{i}^{2}\right) .
$$

\section{Virial theorem}

A different technique for ruling out static solutions under certain assumptions about the potential is to consider the virial theorem.

Calculating the spatial trace $T_{i i}$ of $T_{\mu \nu}$, given by (4.34), in a $d$-dimensional Minkowski background for a purely static field, we find that

$$
T_{i i}=\frac{1}{2}(2 n+1-d)|Y|^{2}-(d-1) V+\text { t.d., }
$$

where t.d. denotes total derivatives, and

$$
\begin{aligned}
& |Y|^{2}=\left(\square^{n / 2} \phi\right)^{2}, \quad n=\text { even; } \\
& |Y|^{2}=\left(\partial_{i} \square^{(n-1) / 2} \phi\right)^{2}, \quad n=\text { odd. }
\end{aligned}
$$

(Because of the assumption that $\phi$ is static, $\square$ is just $\partial_{i} \partial_{i}$ here.) Similarly, the energy density is given by

$$
T_{00}=\frac{1}{2}|Y|^{2}+V+\text { t.d. }
$$

Note that the total energy $\int d^{d-1} x T_{00}$ is always positive provided that $V$ is non-negative.

Because, for a general energy-momentum tensor, $\partial_{i} T_{i j}=0$ for a static field in Minkowski spacetime, we have $\partial_{i}\left(x_{j} T_{i j}\right)=T_{i i}$. Integrating this over the $(d-1)$ dimensional space, and assuming that the falloff conditions for $\phi$ are such that the surface term gives zero, it follows that we must have

$$
\int d^{d-1} x T_{i i}=0
$$

and hence, from (6.9),

$$
\int d^{d-1} x\left[\frac{1}{2}(d-1-2 n)|Y|^{2}+(d-1) V\right]=0 .
$$

(The same result may be obtained by using Derrick's scaling argument [38], and demanding that the total energy $\int d^{d-1} x T_{00}$ be extremized for any static solution.)

We see from (6.13) that if $V$ is positive and $d>2 n+1$, there can be no static solutions. Similarly, if $V$ is negative and $d<2 n+1$, there can again be no static solutions.

\section{Stationary metrics}

The results of Secs. VIA and VIB remain valid for globally stationary metrics; that is, metrics admitting an everywhere timelike Killing vector field $K^{\mu}$. This is because the condition $K^{\mu} \partial_{\mu} \phi=0$ implies that $\partial_{\mu} \phi$ is spacelike, or zero, and so $g^{\mu \nu} \partial_{\mu} \phi \partial_{\nu} \phi \geq 0$. In the case that $K^{\mu}$ is not everywhere timelike, in other words an ergoregion is present, the argument just given will not necessarily be valid. However, if the metric is both stationary and axisymmetric, and $\phi$ is assumed to be both stationary and axisymmetric, the argument will go through.

\section{STABILIZATION BY HUBBLE FRICTION}

Following the work of [39], we have studied the behavior of the Pais-Uhlenbeck oscillator in a background de Sitter universe. We choose coordinates in which the metric is given by 


$$
d s^{2}=-d t^{2}+e^{2 H t} d x_{i} d x_{i},
$$

where $H=\sqrt{\Lambda / 3}$ and $\Lambda$ is the cosmological constant. The nonlinear fourth-order Pais-Uhlenbeck equation of motion for a spatially homogeneous scalar field $\phi(t)$ with a potential $V(\phi)$ is

$$
\left(\frac{d^{2}}{d t^{2}}+3 H \frac{d}{d t}+m_{1}^{2}\right)\left(\frac{d^{2}}{d t^{2}}+3 H \frac{d}{d t}+m_{2}^{2}\right) \phi=-V^{\prime}(\phi) .
$$

In the absence of the potential, we have two uncoupled damped simple harmonic oscillators, with the solution

$$
\phi=\sum_{i=1}^{2} A_{i} e^{-\frac{3}{2} H t} \sin \left(\sqrt{m_{i}^{2}-\frac{9 H^{2}}{4}} t+\alpha_{i}\right),
$$

as long as $m_{i}^{2}>9 H^{2} / 4$.

If we first set $H=0$ and consider, as an example, a potential $V=\frac{1}{4} \lambda \phi^{4}$, we can verify Pavšič's observation [6] that for given initial data, the solution in Minkowski spacetime remains bounded provided that $\lambda$ is sufficiently small. If we choose a larger value of $\lambda$ such that the solution becomes unstable, we can then verify, by numerical analysis, that turning on $H$ can render it stable again, and, in fact, $\phi$ then decays to zero at large $t$ (i.e., within a few Hubble times).

One may also consider inhomogeneous solutions of the free fourth-order equation, with wave vector $\mathbf{k}$. The equation of motion is obtained by replacing $m_{i}^{2}$ by $m_{i}^{2}+$ $\mathbf{k}^{2} e^{-2 H t}$ in (7.2). The additional term decays to zero rapidly, and so we would expect that our results in the nonlinear situation will remain valid. A complete treatment would require further investigation going beyond the ordinary differential equations we studied here. (See also the discussion in [40].)

Our results will be qualitatively similar for all the higherorder Pais-Uhlenbeck oscillators.

\section{CONCLUSION}

In this paper we have studied a scalar field $\phi$ coupled to a fixed background metric $g_{\mu \nu}$, for which the scalar equation of motion contains derivatives of arbitrary order. In all the examples considered, the equations of motion are linear in $\phi$ apart from nonlinearities $V^{\prime}(\phi)$ introduced by a possible potential term $V(\phi)$. It follows that classically the propagation is causal; that is, the characteristic surfaces across which discontinuities may propagate are null hypersurfaces with respect to the metric $g_{\mu \nu}$. This is despite the fact that the various energy-momentum tensors, such as the canonical or the Belinfante-Rosenfeld energy-momentum tensor, do not satisfy the dominant energy condition.

We have obtained general expressions for both the canonical and the symmetric Belinfante-Rosenfeld energymomentum tensors, in the latter case by introducing auxiliary fields. This formulation greatly simplifies the calculations, since it eliminates the need to vary the metrics in high-order covariant derivatives of the scalar field. The Belinfante-Rosenfeld energy-momentum tensor is required for a consistent coupling of the scalar field to the gravitational field. We have also obtained general expressions for the conserved symplectic current used in quantizing the scalar field. Both exhibit the well known difficulty that the energy-momentum tensors will not satisfy the usual energy conditions, and the sesquilinear inner product defined using the symplectic current will not be positive definite.

Thus, if the standard definition of "positive frequency" is adopted, the quantum mechanical norm on states will be indefinite. On the other hand, swapping the sign in the definition of "positive frequency" for the modes carrying negative energy results in physical states carrying negative energy.

To investigate this further we have considered Euclidean formulations of the quantized theory in which the metric $g_{\mu \nu}$ is taken to be positive definite. Assuming that the Riemannian manifold admits a reflection map decomposing the manifold into two disjoint domains symmetric about a separating hypersurface, we have investigated whether Osterwalder and Schrader's reflection positivity condition holds. We show that this is never the case for the theories we study, confirming earlier studies indicating that the Euclidean approach to these theories leads inevitably to the presence of "ghosts," that is, states of negative norm.

Finally, we studied, without assuming spherical symmetry, the possible existence of nonsingular finite energy static solutions of our equations on static or stationary backgrounds, and were able to rule out such solutions subject to various assumptions on the potential $V(\phi)$. These results encompass the important cases of soliton solutions in flat space, bounce solutions inducing the decay of false vacua, and scalar hair for black holes.

Although the implications of higher derivative theories for quantum gravity at the microscopic level remain unclear, the work of $[6-9,14]$ and the cosmological studies of [39] indicate that there is still much to be understood about the behavior of such higher derivative theories at the macroscopic, classical, level. After all, the discoveries of Slipher, Friedmann, Lemaitre, Hubble, and Riess et al. [41-45] clearly indicate that the universe at the largest scale is definitely not in a state of static equilibrium. Moreover, as we illustrated in this paper, the phenomenon of Hubble friction clearly mitigates against the instability, in the expanding state, of modes that are unstable in a static configuration.

\section{ACKNOWLEDGMENTS}

We thank Stam Nicolis, Michael Volkov, and Claude Warnick for informative discussions on higher derivative theories. G. W. G. is grateful for the award of a LE STUDIUM Chair held at the LMPT of the University of 
Tours under the auspices of which some of the work described in this paper was carried out. The work of C. N.P. is supported in part by DOE Grant No. DE-FG0213ER42020. The research of S. S. was partially supported by the ERC-AdG-2015 Grant No. 694896. C. N.P. is grateful to the University of Tours for hospitality during the course of some of this work. S.S. thanks M. Shaposhnikov for warm hospitality at EPFL during some stages of this project.

\section{APPENDIX: CANONICAL NOETHER ENERGY-MOMENTUM TENSOR}

In this Appendix, we discuss the construction of the canonical conserved energy-momentum tensor for a scalar field with a higher derivative equation of motion, in a Minkowski spacetime background. We show also in some example cases how the canonical energy-momentum tensor is related to the conventional energy momentum that is derived by the Belinfante-Rosenfeld procedure of coupling the scalar to gravity and then varying the action with respect to the metric. In particular, we show in these examples how the canonical energy-momentum tensor can be derived by applying the Belinfante-Rosenfeld procedure to an action in which an appropriate nonminimal coupling of the scalar field to gravity has been added.

Consider a scalar field $\phi$ in Minkowski spacetime, described by a Lagrangian

$$
\mathcal{L}=\mathcal{L}\left(\phi, \phi_{\nu_{1}}, \phi_{\nu_{1} \nu_{2}}, \phi_{\nu_{1} \nu_{2} \nu_{3}}, \ldots\right),
$$

where $\phi_{\nu_{1}}=\partial_{\nu_{1}} \phi, \quad \phi_{\nu_{1} \nu_{2}}=\partial_{\nu_{1}} \partial_{\nu_{2}} \phi$, etc. The EulerLagrange equation is

$\frac{\partial \mathcal{L}}{\partial \phi}=\partial_{\nu_{1}} \frac{\partial \mathcal{L}}{\partial \phi_{\nu_{1}}}-\partial_{\nu_{1}} \partial_{\nu_{2}} \frac{\partial \mathcal{L}}{\partial \phi_{\nu_{1} \nu_{2}}}+\partial_{\nu_{1}} \partial_{\nu_{2}} \partial_{\nu_{3}} \frac{\partial \mathcal{L}}{\partial \phi_{\nu_{1} \nu_{2} \nu_{3}}}+\cdots$

Using this to substitute for $\partial \mathcal{L} / \partial \phi$ in

$$
\begin{aligned}
\partial_{\mu} \mathcal{L}= & \frac{\partial \mathcal{L}}{\partial \phi} \phi_{\mu}+\frac{\partial \mathcal{L}}{\partial \phi_{\nu_{1}}} \phi_{\nu_{1} \mu}+\frac{\partial \mathcal{L}}{\partial \phi_{\nu_{1} \nu_{2}}} \phi_{\nu_{1} \nu_{2} \mu}+\frac{\partial \mathcal{L}}{\partial \phi_{\nu_{1} \nu_{2} \nu_{3}}} \phi_{\nu_{1} \nu_{2} \nu_{3} \mu} \\
& +\cdots,
\end{aligned}
$$

then after some straightforward manipulations one finds that

$$
\partial_{\mu} \mathcal{L}=\partial_{\nu} \sum_{n \geq 1} W_{\mu}^{(n) \nu}
$$

and hence that $T_{\mu}{ }^{\nu}$ defined by

$$
T_{\mu}{ }^{\nu}=-\sum_{n \geq 1} W_{\mu}^{(n) \nu}+\delta_{\mu}^{\nu} \mathcal{L}
$$

is conserved, $\partial_{\nu} T_{\mu}{ }^{\nu}=0$, where

$$
\begin{aligned}
W_{\mu}^{(1) \nu}= & \frac{\partial \mathcal{L}}{\partial \phi_{\nu}} \phi_{\mu}, \\
W_{\mu}^{(2) \nu}= & \frac{\partial \mathcal{L}}{\partial \phi_{\alpha_{1} \nu}} \phi_{\alpha_{1} \mu}-\partial_{\alpha_{1}} \frac{\partial \mathcal{L}}{\partial \phi_{\alpha_{1} \nu}} \phi_{\mu}, \\
W_{\mu}^{(3) \nu}= & \frac{\partial \mathcal{L}}{\partial \phi_{\alpha_{1} \alpha_{2} \nu}} \phi_{\alpha_{1} \alpha_{2} \mu}-\partial_{\alpha_{1}} \frac{\partial \mathcal{L}}{\partial \phi_{\alpha_{1} \alpha_{2} \nu}} \phi_{\alpha_{2} \mu} \\
& +\partial_{\alpha_{1}} \partial_{\alpha_{2}} \frac{\partial \mathcal{L}}{\partial \phi_{\alpha_{1} \alpha_{2} \nu}} \phi_{\mu}, \\
W_{\mu}^{(4) \nu}= & \frac{\partial \mathcal{L}}{\partial \phi_{\alpha_{1} \alpha_{2} \alpha_{3} \nu}} \phi_{\alpha_{1} \alpha_{2} \alpha_{3} \mu}-\partial_{\alpha_{1}} \frac{\partial \mathcal{L}}{\partial \phi_{\alpha_{1} \alpha_{2} \alpha_{3} \nu}} \phi_{\alpha_{2} \alpha_{3} \mu} \\
& +\partial_{\alpha_{1}} \partial_{\alpha_{2}} \frac{\partial \mathcal{L}}{\partial \phi_{\alpha_{1} \alpha_{2} \alpha_{3} \nu}} \phi_{\alpha_{3} \mu}-\partial_{\alpha_{1}} \partial_{\alpha_{2}} \partial_{\alpha_{3}} \frac{\partial \mathcal{L}}{\partial \phi_{\alpha_{1} \alpha_{2} \alpha_{3} \nu}} \phi_{\mu},
\end{aligned}
$$

and so on. Note that $-W_{\mu}^{(n) \nu}$ gives the contribution to the canonical energy-momentum tensor associated with the dependence of the Lagrangian on the $n$ th-order spacetime derivatives of $\phi$. As always with the canonically defined energy-momentum tensor coming from the Noether symmetries of the Lagrangian (i.e., the fact that the Lagrangian has no explicit dependence on the spacetime coordinates $\left.x^{\mu}\right), T_{\mu}{ }^{\nu}$ may not necessarily be symmetric in $\mu$ and $\nu$ after lowering the $\nu$ index. In the event that it is not, one can construct a conserved symmetric 2-index tensor from it by making use of the freedom to add a term $\partial_{\sigma} \psi_{\mu}{ }^{\nu \sigma}$ to $T_{\mu}{ }^{\nu}$, where $\psi_{\mu}{ }^{\nu \sigma}$ is any tensor that is antisymmetric in $\nu$ and $\sigma$,

$$
\psi_{\mu}^{\nu \sigma}=-\psi_{\mu}{ }^{\sigma \nu} .
$$

Here we present examples of the canonical energymomentum tensors for some simple Lagrangians. For the standard kinetic term for a scalar field we obtain

$$
\begin{aligned}
\mathcal{L} & =-\frac{1}{2}(\partial \phi)^{2}, \\
T_{\mu}{ }^{\nu} & =\partial_{\mu} \phi \partial^{\nu} \phi-\frac{1}{2} \delta_{\mu}^{\nu}(\partial \phi)^{2},
\end{aligned}
$$

which is the same as the conventional Belinfante-Rosenfeld energy-momentum tensor that one would obtain from varying the metric in $\mathcal{L}=-\frac{1}{2} \sqrt{-g}(\partial \phi)^{2}$ and then specializing to a Minkowski background. If instead we were to integrate the Lagrangian by parts before the calculation of the canonical energy-momentum tensor, we would obtain instead the energy-momentum tensor $\tilde{T}_{\mu_{\nu}}$ :

$$
\begin{aligned}
\mathcal{L} & =\frac{1}{2} \phi \square \phi, \\
\tilde{T}_{\mu}{ }^{\nu} & =-\frac{1}{2} \phi \partial_{\mu} \partial^{\nu} \phi+\frac{1}{2} \partial_{\mu} \phi \partial^{\nu} \phi+\frac{1}{2} \delta_{\mu}^{\nu} \phi \square \phi .
\end{aligned}
$$


The difference between the two is

$$
\tilde{T}_{\mu}{ }^{\nu}-T_{\mu}{ }^{\nu}=\frac{1}{4}\left(\delta_{\mu}^{\nu} \square-\partial_{\mu} \partial^{\nu}\right) \phi^{2},
$$

and, in fact, $\tilde{T}_{\mu}{ }^{\nu}$ is the conventional Belinfante-Rosenfeld energy-momentum tensor one would obtain by varying the metric in the nonminimally coupled Lagrangian

$$
\tilde{\mathcal{L}}=\sqrt{-g}\left[-\frac{1}{2}(\partial \phi)^{2}-\frac{1}{8} R \phi^{2}\right],
$$

and then specializing to the Minkowski spacetime background.

If we consider now a fourth-order Lagrangian, we obtain

$$
\begin{aligned}
\mathcal{L} & =-\frac{1}{2}(\square \phi)^{2}, \\
T_{\mu}^{\prime \nu} & =(\square \phi) \partial_{\mu} \partial^{\nu} \phi-\left(\partial^{\nu} \square \phi\right) \partial_{\mu} \phi-\frac{1}{2}(\square \phi)^{2} \delta_{\mu}^{\nu} .
\end{aligned}
$$

We have denoted this tensor with a prime, because it is not symmetric in $\mu$ and (the lowered) $\nu$ indices. We can obtain a symmetric conserved energy-momentum tensor by adding a term $\partial_{\sigma} \psi_{\mu}{ }^{\nu \sigma}$ with

$$
\psi_{\mu}{ }^{\nu \sigma}=\left(\partial_{\mu} \partial^{\nu} \phi\right) \partial^{\sigma} \phi-\left(\partial_{\mu} \partial^{\sigma} \phi\right) \partial^{\nu} \phi,
$$

leading to the energy-momentum tensor $T_{\mu}{ }^{\nu}=T_{\mu}^{\prime \nu}+$ $\partial_{\sigma} \psi_{\mu}{ }^{\nu \sigma}$, where

$$
\begin{aligned}
T_{\mu}{ }^{\nu}= & 2\left(\partial_{\mu} \partial^{\nu} \phi\right) \square \phi-\left(\partial_{\mu} \phi\right) \square\left(\partial^{\nu} \phi\right)-\left(\partial^{\nu} \phi\right) \square\left(\partial_{\mu} \phi\right) \\
& +\left(\partial_{\mu} \partial^{\nu} \partial_{\alpha} \phi\right)\left(\partial^{\alpha} \phi\right)-\left(\partial_{\mu} \partial^{\alpha} \phi\right)\left(\partial^{\nu} \partial_{\alpha} \phi\right) \\
& -\frac{1}{2} \delta_{\mu}^{\nu}(\square \phi)^{2} .
\end{aligned}
$$

The tensors (A9) and (A14) are precisely of the form of the second-order and fourth-order contributions in the energymomentum tensor (3.12) given in [24] for the fourth-order Pais-Uhlenbeck theory.

The canonical energy-momentum tensor $T_{\mu}{ }^{\nu}$ for the $-\frac{1}{2}(\square \phi)^{2}$ Lagrangian, given by (A14), is different from the flat-space specialization of the expression (4.19) that we obtained by the Belinfante-Rosenfeld metric-variation method. In fact, the difference between the two energymomentum tensors can be generated as the metric variation of a certain nonminimal coupling of the scalar field to gravity. Specifically, we find that the canonical energymomentum tensor in (A14) can be obtained by varying the metric in the Lagrangian

$\tilde{\mathcal{L}}=\sqrt{-g}\left[-\frac{1}{2}(\square \phi)^{2}+\left(R_{\mu \nu}-\frac{1}{4} R g_{\mu \nu}\right) \partial^{\mu} \phi \partial^{\nu} \phi\right]$,

and then specializing to a Minkowski-spacetime background. The nonminimal coupling term in (A15) is similar in form to a Horndeski coupling [46] that has been studied in other contexts in the literature, namely a term $\sqrt{-g}\left(R_{\mu \nu}-\frac{1}{2} R g_{\mu \nu}\right) \partial^{\mu} \phi \partial^{\nu} \phi$.
[1] G. 't Hooft and M. J. G. Veltman, One loop divergencies in the theory of gravitation, Ann. Inst. Henri Poincaré, A 20, 69 (1974).

[2] M. H. Goroff and A. Sagnotti, Quantum gravity at two loops, Phys. Lett. 160B, 81 (1985).

[3] K. S. Stelle, Renormalization of higher derivative quantum gravity, Phys. Rev. D 16, 953 (1977).

[4] A. Pais and G. E. Uhlenbeck, On field theories with nonlocalized action, Phys. Rev. 79, 145 (1950).

[5] M. Ostrogradsky, Mémoires sur les équations différentielles, relatives au problème des isopérimètres, Mem. Acad. St. Petersbourg 6, 385 (1850).

[6] M. Pavšič, Stable self-interacting Pais-Uhlenbeck oscillator, Mod. Phys. Lett. A 28, 1350165 (2013).

[7] A. V. Smilga, Ghost-free higher-derivative theory, Phys. Lett. B 632, 433 (2006).

[8] A. V. Smilga, Benign versus malicious ghosts in higherderivative theories, Nucl. Phys. B706, 598 (2005).

[9] A. V. Smilga, Comments on the dynamics of the PaisUhlenbeck oscillator, SIGMA 5, 017 (2009).
[10] A. Salvio, Metastability in quadratic gravity, Phys. Rev. D 99, 103507 (2019).

[11] N. Boulanger, F. Buisseret, F. Dierick, and O. White, Higher-derivative harmonic oscillators: Stability of classical dynamics and adiabatic invariants, Eur. Phys.J. C 79, 60 (2019).

[12] S. Nicolis, Higher time derivatives in the microcanonical ensemble describe dynamics of flux-coupled classical and quantum oscillators, arXiv:1805.07934.

[13] R. P. Woodard, Ostrogradsky's theorem on Hamiltonian instability, Scholarpedia 10, 32243 (2015).

[14] M. Pavšič, Pais-Uhlenbeck oscillator and negative energies, Int. J. Geom. Methods Mod. Phys. 13, 1630015 (2016).

[15] S. W. Hawking, Who's afraid of (higher derivative) ghosts? in Quantum Field Theory and Quantum Statistics: Essays in honour of sixtieth birthday of E.S. Fradkin, edited by I. A. Batalin, G. A. Vilkovisky, and C. J. Isham (Adam Hilger, Bristol, 1987), Vol. 2, pp. 129-139.

[16] S. W. Hawking and T. Hertog, Living with ghosts, Phys. Rev. D 65, 103515 (2002). 
[17] I. Cho and O. K. Kwon, Generalized Lee-Wick formulation from higher derivative field theories, Phys. Rev. D 82, 025013 (2010).

[18] E. T. Whittaker, A Treatise on the Analytical Dynamics of Particles and Rigid Bodies, 4th ed. (Cambridge University Press, Cambridge, England, 1988).

[19] G. W. Gibbons and J. B. Hartle, Real tunneling geometries and the large scale topology of the universe, Phys. Rev. D 42, 2458 (1990).

[20] K. Osterwalder and R. Schrader, Axioms for Euclidean Green's functions, Commun. Math. Phys. 31, 83 (1973).

[21] E. Massa, S. Vignolo, R. Cianci, and S. Carloni, A new geometrical look at Ostrogradsky's procedure, Int. J. Geom. Methods Mod. Phys. 15, 1850128 (2018).

[22] C. M. Bender and P. D. Mannheim, No-Ghost Theorem for the Fourth-Order Derivative Pais-Uhlenbeck Oscillator Model, Phys. Rev. Lett. 100, 110402 (2008).

[23] A. Salvio and A. Strumia, Quantum mechanics of 4-derivative theories, Eur. Phys. J. C 76, 227 (2016).

[24] K. Andrzejewski, J. Gonera, and P. Maslanka, Euclidean path integral and higher-derivative theories, Prog. Theor. Phys. 125, 247 (2011).

[25] F. J. Belinfante, On the current and the density of the electric charge, the energy, the linear momentum and the angular momentum of arbitrary fields, Physica (Utrecht) 7, 449 (1940).

[26] L. Rosenfeld, Sur le tenseur d'impulsion-energie, Acad. R. Belg. Memoirs Classes Science 18, 1 (1940).

[27] A. Uhlmann, Some remarks on reflection positivity, Czech. J. Phys. B 29, 117 (1979).

[28] A. Uhlmann, On a Riemannian method in quantum theory about curved space-time, Czech. J. Phys. B 31, 1249 (1981).

[29] A. Uhlmann, Riemannian method in quantum field theory about curved space-time, Czech. J. Phys. B 32, 573 (1982).

[30] A. M. Jaffe, S. Klimek, and A. Lesniewski, Representations of the Heisenberg algebra on a Riemann surface, Commun. Math. Phys. 126, 421 (1989).

[31] G. W. Gibbons, Topology change in classical and quantum gravity, in Kyoto 1991, in 6th Marcel Grossman meeting on general relativity: Proceedings, edited by $\mathrm{H}$. Sato and T. Nakamura (World Scientific, Singapore, 1992), pp. 1013-1032.
[32] G. W. Gibbons and H. J. Pohle, Complex numbers, quantum mechanics and the beginning of time, Nucl. Phys. B410, 117 (1993).

[33] A. Jaffe and G. Ritter, Quantum field theory on curved backgrounds. I. The Euclidean functional integral, Commun. Math. Phys. 270, 545 (2007).

[34] A. Jaffe and G. Ritter, Quantum field theory on curved backgrounds. II. Spacetime symmetries, arXiv:0704.0052.

[35] A. Jaffe and G. Ritter, Reflection positivity and monotonicity, J. Math. Phys. (N.Y.) 49, 052301 (2008).

[36] C. C. Anderson, Defining physics at imaginary time: Reflection positivity for certain Riemannian manifolds, Ph.D. thesis, Harvard University, 2015.

[37] W. Pauli and F. Villars, On the invariant regularization in relativistic quantum theory, Rev. Mod. Phys., 21, 434 (1949).

[38] G. H. Derrick, Comments on nonlinear wave equations as models for elementary particles, J. Math. Phys. (N.Y.) 5, 1252 (1964).

[39] G. Pulgar, J. Saavedra, G. Leon, and Y. Leyva, Higher order Lagrangians inspired by the Pais-Uhlenbeck oscillator and their cosmological applications, J. Cosmol. Astropart. Phys. 05 (2015) 046.

[40] N. Deruelle, M. Sasaki, Y. Sendouda, and A. Youssef, Inflation with a Weyl term, or ghosts at work, J. Cosmol. Astropart. Phys. 03 (2011) 040.

[41] A. Friedmann, Über die Krümmung des Raumes, Z. Phys. 10, 377 (1922).

[42] G. Lemaître, Un univers homogène de masses constant et de rayon croissant, rendant compte de la vitesse radiale des nèbuleuses extra-galactiques, Ann. Soc. Sci. Bruxelles A 47, 49 (1927).

[43] V. Slipher, Spectrographic observations of nebulae, Popular Astronomy 23, 21 (1915).

[44] E. Hubble, A relation between distance and radial velocity among extra-galactic nebulae, Proc. Natl. Acad. Sci. U.S.A. 15, 168 (1929).

[45] A. G. Riess et al., Observational evidence from supernovae for an accelerating universe and a cosmological constant, Astron. J. 116, 1009 (1998).

[46] G. W. Horndeski, Second-order scalar-tensor field equations in a four-dimensional space, Int. J. Theor. Phys. 10, 363 (1974). 